\title{
The competitive facility location problem under disruption risks
}

\begin{abstract}
Two players sequentially locate a fixed number of facilities, competing to capture market share. Facilities face disruption risks, and each customer patronizes the nearest operational facility, regardless of who operates it. The problem therefore combines competitive location and location with disruptions. This combination has been absent from the literature. We model the problem as a Stackelberg game in which the leader locates facilities first, followed by the follower, and formulate the leader's decision problem as a bilevel optimization problem. A variable neighborhood decomposition search heuristic which includes variable fixing and cut generation is developed. Computational results suggest that high quality solutions can be found quickly. Interesting managerial insights are drawn.
\end{abstract}

Keywords: competitive location; facility disruptions; bilevel optimization; local search; variable neighborhood search

\section{Introduction}

This paper introduces the competitive facility location problem under disruption risks (CFLPD), a discrete facility location model that, to the best of our knowledge, is the first to incorporate possible disruptions into a competitive facility location problem. In many industries, service competition is present among multiple firms such as supermarkets or gas stations. Customers may choose among competing facilities based on distance (as we assume in this paper), quality, brand loyalty, or other factors. In addition, facilities may face disruptions from time to time due to natural disasters, labor actions, or power outages. When a facility is disrupted, its customers may seek service from another operational facility belonging to the same player; they may seek service from a facility belonging to a different player; or their sales may be lost entirely. In either of last two cases, the customer's original service provider loses revenue, and in all three cases, the customers incur higher service costs. For express carriers such as FedEx and UPS, both service competition and delivery delays or labor disputes will influence their brand recognition, service quality and market share. For example, a FedEx store may lose its customers if it delivers packages late due to labor actions or other disruptions, or if a UPS store is nearby. This highlights the need for an optimal facility deployment that considers both service competition and probabilistic facility disruptions. 
We consider a supplier-receiver network with multiple customers and two noncooperative firms (the players of the Stackelberg game): the leader and the follower. The players make facility location decisions sequentially, with each aiming to maximize its own market share or revenue. This setup is well modeled as a Stackelberg game (Dempe 2002) in which each player has exactly one move. The leader will first open $B$ facilities, anticipating that the follower will react rationally by optimally placing $K$ facilities. Each customer has a demand and seeks the nearest operating facility for service. We assume a binary preference model in which each customer chooses only a single operating facility for service at any one time.

In addition, the facilities opened are subject to disruptions. When a facility is disrupted, it cannot serve customers. Following Snyder and Daskin (2005), we assume that customers of disrupted facilities are reassigned to another (functional) facility. In particular, we assign each customer to multiple facilities in a sequence of assignment levels $r=1,2, \cdots 1$ For each customer, the closest facility $(r=1)$, the so-called the primary facility, will serve it under normal circumstances. If the primary facility fails, the customer is served by its first backup facility $(r=2)$. If that facility fails too, it is served by its level-3 facility, and so on. In general, a facility assigned to a customer at level $r$ serves that customer if all $r-1$ facilities at lower levels have failed. If a customer's primary facility fails and the nearest operating facility is owned by the other player, then the player that owns the primary facility will lose that customer until the disruption ends. If all of the facilities assigned to the customer are disrupted, the customer is lost by both of the players.

We formulate the problem as a binary bilevel linear optimization problem (BBLP). The model determines the optimal locations for the leader in order to maximize her market share, under the strongest possible response by the follower. If the facilities are assumed to be always reliable, i.e., each customer can always be captured by the nearest facility, then we obtain as a special case the discrete $(r \mid p)$-centroid problem (RPCP) (Alekseeva et al. 2010) and the closely related competitive maximal covering location model (Serra and ReVelle 1994, Seyhan et al. 2015). (The competitive maximal covering model assumes that customers will only patronize facilities within a given coverage radius, whereas the RPCP allows any assignment, regardless of distance; otherwise, the two problems are identical.)

It has been shown that the discrete RPCP is NP-hard; in fact, it belongs to the class of $\sum_{2}^{P}$-hard problems (Noltemeier et al. 2007). This means that to check whether a (leader's) decision is feasible requires solving an NP-hard problem (to optimize the follower's strategy). This study addresses this

\footnotetext{
${ }^{1}$ Note that we index the levels beginning at $r=1$, whereas Snyder and Daskin $(2005)$ and others index them beginning at $r=0$.
} 
complicated but also realistic problem. Our main contributions are as follows: First, we construct a BBLP model for this new type of facility location problem. Second, we develop a matheuristic based on variable neighborhood decomposition search (VNDS) which includes variable fixing and cut generation interactively. We further show that this matheuristic can be extended to a large class of BBLP directly. Third, extensive experiments and sensitive analysis demonstrate the effectiveness of our approach. Results on RPCP benchmarks show that the VNDS matheuristic is very promising compared to the current best heuristics and exact approaches for this special case. Results on the more general CFLPD instances draw many interesting managerial insights on the approximate facility deployment strategies and market share competition.

The remainder of this paper is organized as follows. We review the relevant literature in $§ 2$ and formulate the CFLPD as a BBLP in $₫ 3$. A matheuristic using VNDS is provided in $\$ 4$. The numerical experiment design and computational results are presented in $\$ 5$. Finally, 86 concludes the paper and discusses future research.

\section{Literature Review}

Facility location problems have been extensively studied in the past few decades, due to the wide variety of applications that arise in placing distribution centers, warehouses, gas stations, and fire stations, as well as in constructing communication networks, and so on. Two of the most well-studied problems are the $p$-median problem and the maximal covering location problem. The $p$-median problem is to locate $p$ facilities so that the total demand-weighted distance between each customer and the nearest facility is minimized. The maximal covering location problem seeks to locate a fixed number (e.g., $p$ ) of facilities so that the number of covered demands is maximized. For overviews of these two classical models, see, for example, Snyder (2010) or Daskin (2013).

Both the $p$-median problem and the maximal covering location problem ignore the effect of competition on the location decision and assume that there is a single decision-maker. However, many firms face location-based competition, and failing to account for this competition when choosing facility locations can result in lower than anticipated market share. Competitive facility location problems take this competition into account; most assume there are two players who successively open their facilities, each aiming to capture customers and maximize revenue. For reviews of competitive location models, see Eiselt et al. (1993), Serra et al. (1994), Eiselt and Laporte (1997), Kress and Pesch (2012) and Farahani et al. (2014). 
One competitive location problem, the RPCP, has attracted increased attention in the past five years. In this problem, the leader places $p$ facilities on a graph knowing that the follower will react by placing $r$ facilities.2. The goal of both the leader and the follower is to maximize its own market share (Alekseeva et al. 2010). This problem is often formulated as a BBLP. Recent algorithms have been tested using instances with up to 100 customers, 100 potential facilities and $p=r=20$ or 30 from the Discrete Location Problems benchmark library33, Exact approaches-including the iterative exact method by Alekseeva et al. (2010), the branch-and-cut (RP-B\&C) method by Roboredo and Pessoa (2013) and the modified iterative exact method (MEM) by Alekseeva and Kochetov (2013) - guarantee global optimality but are very computationally intensive (e.g., more than 10 hours for $p=r=10$; see Roboredo and Pessoa (2013)). The iterative exact method by Alekseeva et al. (2010) is based on a single-level binary optimization problem with exponentially many constraints and variables. The model is repeatedly solved, with new sets of follower locations added iteratively. The RP-B\&C method by Roboredo and Pessoa (2013) is similar but with only a polynomial number of variables, and the exponentially many constraints are lifted into stronger inequalities. MEM (Alekseeva and Kochetov 2013 improves the iterative exact method by using a model with only a polynomial number of variables and by using the strengthening inequalities introduced by Roboredo and Pessoa (2013). Heuristics based on operations (e.g., probabilistic swapping) for the p-median problem have shown high accuracy for a relatively small number of iterations. However, the complexity of each iteration still remains rather high, because the follower's problem has to be solved to evaluate the leader's neighborhood solution. The most effective heuristic methods proposed to date are the hybrid memetic algorithm (HMA) by Alekseeva et al. (2009), the tabu search algorithm with Lagrangian relaxation (TSL) by Davydov (2012), the variable neighborhood search (VNS) and stochastic tabu search (STS) by Davydov et al. (2014), and the hybrid genetic algorithm with solution archive (GA+solA) by Biesinger et al. (2015).

As noted above, the RPCP is closely related to the competitive maximal covering location problem, introduced by Serra and ReVelle (1994) and based on the classical maximal covering location problem (Church and Velle 1974). Most heuristics for this problem, including the heuristic by Serra and ReVelle (1994), involve iterating between the leader's and follower's decisions, which will result in finding a Nash equilibrium (NE) but not necessarily the NE that is best for the leader. For the same problem

${ }^{2}$ When discussing the RPCP, we will use $p$ and $r$ to denote the number of facilities opened by the leader and the follower, respectively, instead of $B$ and $K$ as in our model, to remain consistent with the existing research.

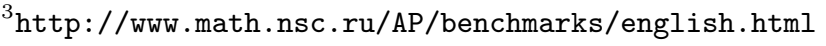


and two variants, Plastria and Vanhaverbeke (2008) restrict the follower to opening a single facility $(K=1)$, which allows them to reformulate the problem as a single-level problem. Seyhan et al. (2015) expand this idea to allow general $K$ by assuming that the follower solves his problem using a greedy algorithm; the greedy solution is captured by a polynomial number of constraints in the leader's single-level problem.

The competitive location literature generally assumes that all facilities are completely reliable. In contrast, facility location models with disruptions have appeared only more recently. Snyder and Daskin (2005) introduce the reliable fixed-charge location problem (RFLP) and reliable $p$-median problem (RPMP), in which facilities are subject to random disruptions with identical probability, to investigate the effect of probabilistic facility failures on the optimal facility deployment. Berman et al. (2007) consider a $p$-median problem under facility disruptions and study its asymptotic properties. Cui et al. (2010) and Aboolian et al. (2012) generalize the work of Snyder and Daskin (2005) by allowing sitedependent disruption probabilities. Zhang et al. (2016) allow site-dependent probabilities and also incorporate inventory costs. Shen et al. (2011) formulate the RFLP as a nonlinear integer optimization problem and propose a near-optimal heuristic for the special case with identical probabilities. See Snyder et al. (2015) for a recent review of the literature on facility location models with disruptions. Note, however, that all of the reliable location models we are aware of assume only a single firm, ignoring the potential competition.

Another related body of literature is that on the network interdiction problem. As in the RPCP and CFLPD, this problem is usually modeled as a Stackelberg game between an intelligent attacker and a defender (Aksen et al. 2014). The attacker's (leader's) objective is to cause the maximum (worst-case) disruption in an existing service network; the defender (follower) is responsible for locating/relocating facilities as well as protecting some of those to guarantee service. The majority of the research has its roots in military, homeland security applications or critical infrastructure planning (O' Hanley and Church 2011, Liberatore et al. 2011, Losada et al. 2012, Gedik et al. 2014). Although they are similar in some respects to facility location problems, network interdiction problems are generally beyond the scope of competitive location problems because only one player's decision involves location/relocation actions.

Finding the optimal facility location design under service competition and disruption risks is extremely hard. Simultaneously using a bilevel optimization structure and a probabilistic facility disruption mechanism makes this problem very challenging to handle, especially for large instances. The bilevel structure introduces a nonconvex and combinatorial nature (Dempe 2002), while disruption 
risks give rise to a large number of probabilistic facility failure scenarios. To the best of our knowledge, only Wang and Ouyang (2013) simultaneously consider spatial competition and facility disruption risks in the context of facility location. They build a leader-follower Stackelberg competition model and design a continuous approximation scheme to find closed-form analytical solutions. In their work, location decisions are represented using continuous and differentiable density functions. Our paper, on the other hand, seeks to optimize facility locations on a discrete network. Our model is, to the best of our knowledge, the first to consider both service competition and disruptions in the context of a discrete facility location problem.

\section{Model Formulation}

In this section, we first introduce the notation that will be used throughout the paper. We then formulate the problem as a BBLP. Finally, we will show how the proposed model can be simplified to suit the RPCP case, thus leading to some cutting planes which will be used in 4 .

\section{$3.1 \quad$ Notation}

\subsubsection{Parameters}

Let $I$ be a set of customer locations and $J$ a set of potential facility locations. The parameter $d_{i j}$ defines the distance between customer $i \in I$ and facility $j \in J$. Associated with each customer $i$ is a demand (or purchasing power) $\mu_{i}$. Each facility in $J$ can fail independently with an equal probability $q$. Each customer can be assigned to up to $R$ facilities - one primary facility and $R-1$ backup facilities - where

$R$ is a constant, $1 \leq R \leq B+K$. To avoid ties in case of equal distances, we assume that two players cannot open the same site, and $d_{i j} \neq d_{i k}, \forall i \in I, j, k \in J, j \neq k$. (If the true distances do have ties, the distances can be perturbed slightly to break them arbitrarily.) The goal of the leader is to choose $B$ locations from $J$ in order to maximize her market share under the assumption that the follower in turn chooses $K$ facilities to maximize his own market share.

To summarize, we have the following parameters:

- $I=$ set of customers, indexed by $i$;

- $J=$ set of potential facility locations, indexed by $j$;

- $R=$ number of assignment levels;

- $\mu_{i}=$ demand of customer $i \in I$; 
- $q=$ (identical) failure probability of each facility;

- $B, K=$ number of facilities that the leader, follower will open;

- $d_{i j}=$ distance between customer $i$ and facility $j$;

- $a_{i j k}=1$ if $d_{i j}<d_{i k}$, for $i \in I, j, k \in J, 0$ otherwise.

Note that if the customers' preferences are based on something other than distance, we can simply redefine $d_{i j}$ as customer $i$ 's "penalty" for using facility $j$, where smaller values indicate stronger preferences.

Realistically, facility failure probabilities may be heterogeneous and may depend, for example, on the facilities' geographical locations. Furthermore, a customer may be more willing to change to another facility if the open facilities are closer, i.e., $R$ could be increasing in $K$ and $B$. Or, different customers may accept a different number of facilities for service. However, for simplicity, in this paper, we assume that the failure probabilities are identical and that $R$ is constant, independent of $K$ and $B$, among all customers. Furthermore, customers may use alternate decision rules, such as the Huff rule Ashtiani et al. 2013), which defines an attractiveness function on the facilities. However, the Huff rule would introduce nonlinearity, so we assume a binary preference for simplicity.

\subsubsection{Decision Variables}

We introduce the following binary decision variables:

$$
\begin{aligned}
& x_{j}= \begin{cases}1, & \text { if facility } j \text { is opened by the leader } \\
0, & \text { otherwise }\end{cases} \\
& y_{j}= \begin{cases}1, & \text { if facility } j \text { is opened by the follower } \\
0, & \text { otherwise }\end{cases} \\
& z_{i r}= \begin{cases}1, & \text { if customer } i \text { is assigned to a leader facility at level } r \\
0, & \text { if customer } i \text { is assigned to a follower facility at level } r\end{cases} \\
& w_{i j r}= \begin{cases}1, & \text { if customer } i \text { is assigned to facility } j \text { at level } r \\
0, & \text { otherwise }\end{cases}
\end{aligned}
$$




\subsection{Bilevel Formulation}

Due to the independent-failure assumption, the probability that a customer receives service from its level- $r$ facility is $(1-q) q^{r-1}$. The players want to maximize their own expected demand captured in the end of the game, taking all possible disruptions into account. The leader's objective function is

$$
\max \sum_{i \in I} \sum_{r=1}^{R} \mu_{i} z_{i r}(1-q) q^{r-1},
$$

while the follower's objective function is

$$
\max \sum_{i \in I} \sum_{r=1}^{R} \mu_{i}\left(1-z_{i r}\right)(1-q) q^{r-1} .
$$

Since this is a zero-sum game, (2) is equivalent to

$$
\min \sum_{i \in I} \sum_{r=1}^{R} \mu_{i} z_{i r}(1-q) q^{r-1} .
$$

Now the discrete CFLPD problem can be formulated as a BBLP model:

$$
\max \sum_{i \in I} \sum_{r=1}^{R} \mu_{i} z_{i r}(1-q) q^{r-1}
$$

s.t.

$$
\begin{aligned}
& \sum_{j \in J} x_{j}=B \\
& x_{j} \in\{0,1\} \quad \forall j \in J
\end{aligned}
$$

where given the leader's decision $\hat{x}, z$ is a component of the optimal solution to the follower's problem:

$$
\min \sum_{i \in I} \sum_{r=1}^{R} \mu_{i} z_{i r}(1-q) q^{r-1}
$$

s.t.

$$
\begin{aligned}
& \sum_{j \in J} y_{j}=K \\
& \hat{x}_{j}+y_{j} \leq 1 \quad \forall j \in J \\
& \hat{x}_{j}+1 \geq z_{i r}+w_{i j r} \quad \forall i \in I, j \in J, 1 \leq r \leq R \\
& y_{j}+z_{i r} \geq w_{i j r} \quad \forall i \in I, j \in J, 1 \leq r \leq R \\
& \sum_{k \in J} a_{i k j}\left(\hat{x}_{k}+y_{k}\right)-\left(1-w_{i j r}\right) M_{r} \leq r \quad \forall i \in I, j \in J, 1 \leq r \leq R
\end{aligned}
$$




$$
\begin{aligned}
& \sum_{j \in J} w_{i j r}=1 \quad \forall i \in I, 1 \leq r \leq R \\
& \sum_{r=1}^{R} w_{i j r} \leq 1 \quad \forall j \in J, i \in I \\
& y_{j}, w_{i j r} \in\{0,1\}, 0 \leq z_{i r} \leq 1 \quad \forall i \in I, j \in J, 1 \leq r \leq R
\end{aligned}
$$

Constraints $(9)$ prevent the players from opening facilities at the same site. Constraints 10 and (11) ensure that if customer $i$ is assigned to facility $j$ at level $r$ (i.e., $w_{i j r}=1$ ), and if $j$ is a leader facility (i.e., $z_{i r}=1$ ), then the leader must open it (i.e., $x_{j}=1$ ); otherwise, if $j$ is a follower facility (i.e., $z_{i r}=0$ ), then the follower must open it (i.e., $y_{j}=1$ ). Summing (10) and (11) results in the following valid inequality which will tighten the linear relaxation:

$$
\hat{x}_{j}+y_{j}+1 \geq 2 w_{i j r} \quad \forall i \in I, j \in J, 1 \leq r \leq R
$$

Constraints 12 guarantee that customers are assigned to open facilities level by level in increasing order of distance. A customer $i$ can't be assigned to facility $j$ at level $r$ if there are more than $r$ closer open facilities. The quantity $\sum_{k \in J} a_{i k j}\left(\hat{x}_{k}+y_{k}\right)$ represents the number of open facilities that are closer than $j$ for customer $i$. $M_{r}=B+K-r$ is a large number.

Finally, constraints (13) say that each customer has to be assigned to a facility at each level (since we assume that customer demands are lost only if all assigned facilities have been disrupted), and constraints (14) prohibit a customer from being assigned to a given facility at more than one level. Constraints (6) and (15) are standard integrality constraints. We can drop the integrality requirements on the $z$ variables; in an optimal solution they will equal 0 or 1 .

From the leader's perspective, (4)-(15) can be viewed as a mathematical optimization problem with a constraint region that is implicitly defined by the follower's subproblem (7)-(15). Once the vector $x$ is chosen, though, the follower simply faces a single-level optimization problem. The upper level (4) - (6) is called the leader's problem; the lower level (7)-(15) is called the follower's problem. Removing the lower-level optimality constraints yields the high point problem (Bialas and Karwan 1984): (4)-(6) and (8) -15 .

\subsection{Remarks}

The proposed CFLPD model reduces to the RPCP when $q=0$ (so that $R=1$ ). To formulate the leader's problem in the RPCP, the subscript $r$ could be removed from variables $w$ and $z$, and constraints

\footnotetext{
${ }^{4}$ We would like to express our gratitude to the referees for the introduction of this inequality.
} 
(14) can be removed since they would be redundant. Constraints 12 can be simplified to

$$
w_{i k} \leq 2-a_{i j k}-\left(\hat{x}_{j}+y_{j}\right) \quad \forall i \in I ; k, j \in J
$$

However, in this case, the follower's problem has a tighter formulation. Given leader's solution $\hat{x}$, we

can define the set of facilities that allow the follower to capture customer $i$ Alekseeva and Kochetov 2013):

$$
J_{i}(\hat{x})=\left\{j \in J \mid d_{i j}<\min _{l \in J}\left\{d_{i l} \mid \hat{x}_{l}=1\right\}\right\} .
$$

Now the follower's problem can be formulated as:

$$
\min \sum_{i \in I} \mu_{i} z_{i}
$$

s.t.

$$
\begin{aligned}
& \sum_{j \in J} y_{j}=K \\
& 1-z_{i} \leq \sum_{j \in J_{i}(\hat{x})} y_{j} \quad \forall i \in I \\
& y_{j} \in\{0,1\}, 0 \leq z_{i} \leq 1, \forall i \in I, j \in J
\end{aligned}
$$

For the RPCP, formulation (19)-(22) has fewer variables and constraints than (7)-(15), which will reduce the computational burden when checking the feasibility of a given leader's solution. For both the CFLPD and RPCP, in order to calculate the leader's objective function, we need to solve the follower's problem, which is NP-hard in the strong sense. Some bilevel methods which solve both the leader's and the follower's problems heuristically produce only what are called semi-feasible solutions (Alekseeva and Kochetov 2013). However, the hybridization of heuristics for the upper level with exact approaches for the lower level allows one to find optimal or near-optimal feasible solutions. The method in $\S 4$ is based on this idea.

\section{Variable Neighborhood Decomposition Search}

Solving a bilevel optimization problem, even in its simplest form, is a difficult task. The vast majority of the methods proposed for bilevel problems have been restricted to special cases, such as using the KKT conditions to transform linear bilevel optimization problems into equivalent single-level ones (e.g., Audet et al. (2007)). This can be implemented by relaxing the integrality restrictions in the follower's 
problem and taking the inner dual to convert the bilevel problem into a single-level maximization problem. If the LP bound is tight, it is beneficial to use these bounds. However, our follower's problem has the variable $w$, which makes the LP relaxation very weak: all customers will be assigned at all levels to the follower as long as $K \geq R$.

Based on the analysis above, we solve the problem as a bilevel one. No practical methods are currently available that can consistently produce optimal solutions for general bilevel problems in reasonable computation time. Moore and Bard (1990) and DeNegre and Ralphs (2009) propose a branch-andbound algorithm and a branch-and-cut algorithm for generalized BBLPs, respectively. According to DeNegre and Ralphs (2009), an integer point $(\bar{x}, \bar{y})$ is bilevel feasible if after fixing the leader's solution $\bar{x}$, solving the follower's problem to optimality does result in the follower's decision $\bar{y}$. With regards to a bilevel infeasible solution $(\hat{x}, \hat{y})$, Moore and Bard (1990) propose to branch on the variable $\hat{x}$ (even if it is integer), while DeNegre and Ralphs (2009) propose to use valid inequalities to separate $(\hat{x}, \hat{y})$.

However, these methods may require substantial enumeration and also require repeated solution of binary mixed integer optimization problems in checking bilevel feasibility of integer solutions. Therefore, direct application can be time-consuming for large instances, so we now focus on a reduced solution space in this section, by fixing some variables and by introducing new linear constraints.

In recent years, a growing literature has proposed heuristics for general MIPs, especially 0-1 MIPs, that combine aspects of local search with mathematical optimization techniques. Such heuristics are known as matheuristics and include the local branching paradigm (Fischetti and Lodi 2003), relaxation

induced neighborhood search (Danna et al. 2005), variable neighborhood search branching (Hansen et al. 2006) and variable neighborhood decomposition search (VNDS) (Lazić et al. 2010, Hanafi et al. 2014), among others. These papers demonstrate that combining heuristic frameworks with the use of generic solvers can often give significant improvements in the resolution of large general MIPs. In this section, we propose a VNDS matheuristic to efficiently solve the CFLPD and show its extendibility to general BBLPs. Some key procedures are elaborated first, followed by the main algorithm.

\subsection{Algorithm Schematic}

Once the facilities open by the players are given, the customer-assignment variables $(w$ and $z)$ can be determined by the customers' proximity to these open facilities. For the simplicity of notation, we will use $\mathcal{X}$ and $\mathcal{Y}$ to represent the set of facilities opened by the leader and the follower, respectively. Recall that $x$ and $y$ are the incidence vectors in formulation (4)-15. Then, $\mathcal{X}=\left\{j \mid x_{j}=1\right\}, \mathcal{Y}=\left\{j \mid y_{j}=1\right\}$. In addition, in the algorithm procedures, $\left(x^{0}, y^{0}\right)$ represents the incumbent solution whose neighborhood 
we are interested in, while $\left(x^{*}, y^{*}\right)$ stores the current global best solution.

Assume that the total "available" demand is $D=\sum_{i \in I} \sum_{r=1}^{R} \mu_{i}(1-q) q^{r-1}$. Given the leader's decision $x^{\prime}$ and the follower's decision $y^{\prime}$, function $\operatorname{COST}\left(x^{\prime}, y^{\prime}\right)$ will return the leader's objective value (demand captured). We will use $f(z)=\sum_{i \in I} \sum_{r=1}^{R} \mu_{i} z_{i r}(1-q) q^{r-1}$ to represent the objective function of the high point problem.

In the sections that follow, given a leader's solution $\bar{x}$, any time we solve the follower's problem exactly by $\bar{y}=$ FOLLOWER-SOLVE $(\bar{x})$, we will have a bilevel feasible solution $(\bar{x}, \bar{y})$. To avoid costly and unnecessary re-evaluations and prevent the search from coming back to the previously visited solutions, we add the following tabu cut,

$$
\sum_{j \in \overline{\mathcal{X}}} x_{j} \leq B-1
$$

where $B$ is the cardinality of the binary support of the leader's solution, to prohibit a revisit to $\bar{x}$.

The Local Search procedure proposed in 4.5 is developed to find the local optimum of the neighborhood around $(\bar{x}, \bar{y})$. Once the local search is terminated and the solution $(\bar{x}, \bar{y})$ is recorded, it is valid to use (23) to cut off any solution $(\bar{x}, \hat{y})$. However, the bilevel feasible solution $(\bar{x}, \bar{y})$ is cut off as well. During the local search, this may cause problems in practice when using a commercial framework, such as Gurobi or CPLEX, since it may prevent the solution from being identified as feasible. Instead, we propose the following cut (24), which is satisfied by $(\bar{x}, \bar{y})$, but not by the combination of $\bar{x}$ with a different follower's solution.

Proposition 1 Given a bilevel feasible solution $(\bar{x}, \bar{y})$ to the high point problem (4) -(6), (8) -(15), let $\mathcal{X}_{0}=\left\{j \mid \bar{x}_{j}=0\right\}, \mathcal{X}_{1}=\left\{j \mid \bar{x}_{j}=1\right\}$ and $\mathcal{Y}_{0}=\left\{j \mid \bar{y}_{j}=0\right\}, \mathcal{Y}_{1}=\left\{j \mid \bar{y}_{j}=1\right\}$. The inequality

$$
\sum_{j \in \mathcal{Y}_{0}} y_{j}+\sum_{j \in \mathcal{Y}_{1}}\left(1-y_{j}\right) \leq K\left(\sum_{j \in \mathcal{X}_{0}} x_{j}+\sum_{j \in \mathcal{X}_{1}}\left(1-x_{j}\right)\right)
$$

is valid for $(\bar{x}, \bar{y})$, and is violated by every other integer solution $(\bar{x}, \hat{y})$.

Proof. Suppose $x=\bar{x}, y=\bar{y}$; then the left- and right-hand sides of (24) are both equal to 0, so the inequality holds and is valid for $(\bar{x}, \bar{y})$.

On the other hand, suppose $x=\bar{x}, y=\hat{y} \neq \bar{y}$; then the left-hand side of (24) is greater than or equal to 1 , while the right-hand side equals 0 , so the inequality is violated.

Fischetti and Lodi (2003) observe that the neighborhood of a feasible 0-1 MIP solution often contains better solutions. To reduce the neighborhood of $(\bar{x}, \bar{y})$, the following local branching cut is added to 
the high point problem,

$$
\Delta(x, \bar{x})+\Delta(y, \bar{y})=\sum_{j \in \overline{\mathcal{X}}}\left(1-x_{j}\right)+\sum_{j \in \overline{\mathcal{Y}}}\left(1-y_{j}\right) \leq r h s
$$

where $r h s$ is the neighborhood size. Cut 25) is further equivalent to $\sum_{j \in \overline{\mathcal{X}}} x_{j}+\sum_{j \in \overline{\mathcal{Y}}} y_{j} \geq B+K-r h s$. As explained by Fischetti and Lodi (2003), given the incumbent solution $(\bar{x}, \bar{y})$, the solution space associated with the current branching node can be partitioned by means of the disjunction

$$
\Delta(x, \bar{x})+\Delta(y, \bar{y}) \leq \text { rhs (left branch) } \quad \text { or } \quad \Delta(x, \bar{x})+\Delta(y, \bar{y}) \geq \text { rhs }+1 \text { (right branch) }
$$

Each left-branch subproblem constrained by $\Delta(x, \bar{x})+\Delta(y, \bar{y}) \leq r h s$ (so-called soft variable fixing) will be easier to solve than the original problem; once it is solved to optimality or proved to contain no better solutions, the right-branch subproblem takes over and a new local branching cut is added.

Also note that in all pseudo-codes, right after an initial solution with objective value $\overline{L B}$ is obtained, an objective cut

$$
f(z) \geq \overline{L B}+\varepsilon
$$

is added to the high point problem; while each time the best lower bound $\overline{L B}$ is improved, the right hand side of the objective cut (26) should be set to the new value. $\varepsilon>0$ is a small real number.

\subsection{Intensification and Diversification}

We first introduce the intensification and diversification procedure, which either intensifies or diversifies a given solution depending on the input parameters. The procedure is presented in Algorithm 1 .

As pointed out by Hansen et al. (2010), for many combinatorial optimization problems, local optima with respect to one or several neighborhoods are located rather close to each other. This implies that a local optimum often provides some information about the global optimum. Based on this fact, the search region may be reduced by using information about local optima that have already been found. Alekseeva and Kochetov (2013) show that the follower's facilities are often in the immediate vicinity of the leader's facilities. The follower attempts to "drag" customers from the leader. Due to this property, and similar to the neighborhoods Fswap and Nswap designed by Davydov et al. (2014), Steps 28 in Algorithm 1 make an attempt to predict the follower's behavior and find the strongest preventive move for the leader. These steps attempt to find all solutions of the leader that result from $x^{0}$ by closing one facility $j$ and opening another facility $k$. This new facility $k$ should be either one from the follower's 
decision $y^{0}$, or one located at no more than the 8 th facility nearest to $j$. This replacement constitutes a new leader solution $x^{1}$.

To evaluate $x^{1}$, the corresponding follower's subproblem has to be solved. Since $x^{1}$ is just an intermediate solution candidate, we are only interested in an approximate objective value for $x^{1}$; the exact value will be too time consuming to solve since the follower's problem is NP-hard. For the $(r \mid p)$ centroid problem, some papers (Biesinger et al. 2015, Davydov et al. 2014) solve the LP relaxation of the follower's problem exactly. This approximation yields a lower bound on the true objective value of $x^{1}$. However, the LP relaxation of our follower's formulation is very weak, as explained in the front of \$4. So instead, we adopt a greedy algorithm for solving the follower's problem, which yields an upper bound on the objective value of $x^{1}$. Follower facilities are placed one after the other according to the following greedy criterion: at each iteration, the facility that can capture the largest demand is opened. This process is repeated until $K$ locations $\left(y^{1}\right)$ are chosen.

Steps 28 of Algorithm 1 will find the best 1-exchange neighborhood for the leader. Steps $9 \sqrt{15}$ will further randomly choose a subset of $B$ facilities from the union set $\mathcal{X}^{0} \cup \mathcal{Y}^{0}$ that will induce the largest approximate objective value for the leader if the follower uses the greedy method. Note that in Step 3 and Step 10, the leader's new decision $x^{1}$ should not have been tabu yet. Finally, as shown in Steps 18 20, if the input parameter is AlwaysUpdated = TRUE, this procedure will always update the incumbent $\left(x^{0}, y^{0}\right)$ (thus a diversification); on the other hand, if isAlwaysUpdated = FALSE, this procedure will update $\left(x^{0}, y^{0}\right)$ only if the new solution $\left(x^{\prime}, y^{\prime}\right)$ is better (thus an intensification).

\subsection{Initialization}

For the initial solution, we select an approximate solution of the $p$-median problem with a weighted distance matrix $\left(\mu_{i} d_{i j}\right)$. In this case, the leader ignores the follower but tries to place her facilities as close to customers as possible. Alekseeva and Kochetov (2013) indicate that this approach yields a good approximate solution. Then, as introduced in Section 4.1, we add the tabu cut with respect to $x^{0}$ to avoid revisiting the same solution. Simultaneously, we will search the neighborhood of the incumbent solution $\left(x^{0}, y^{0}\right)$ to see whether it can be further improved by the procedure "Mutation". Finally, we add the objective cut to discard worse solutions in the future search. The initialization procedure is illustrated in Algorithm 2 . 

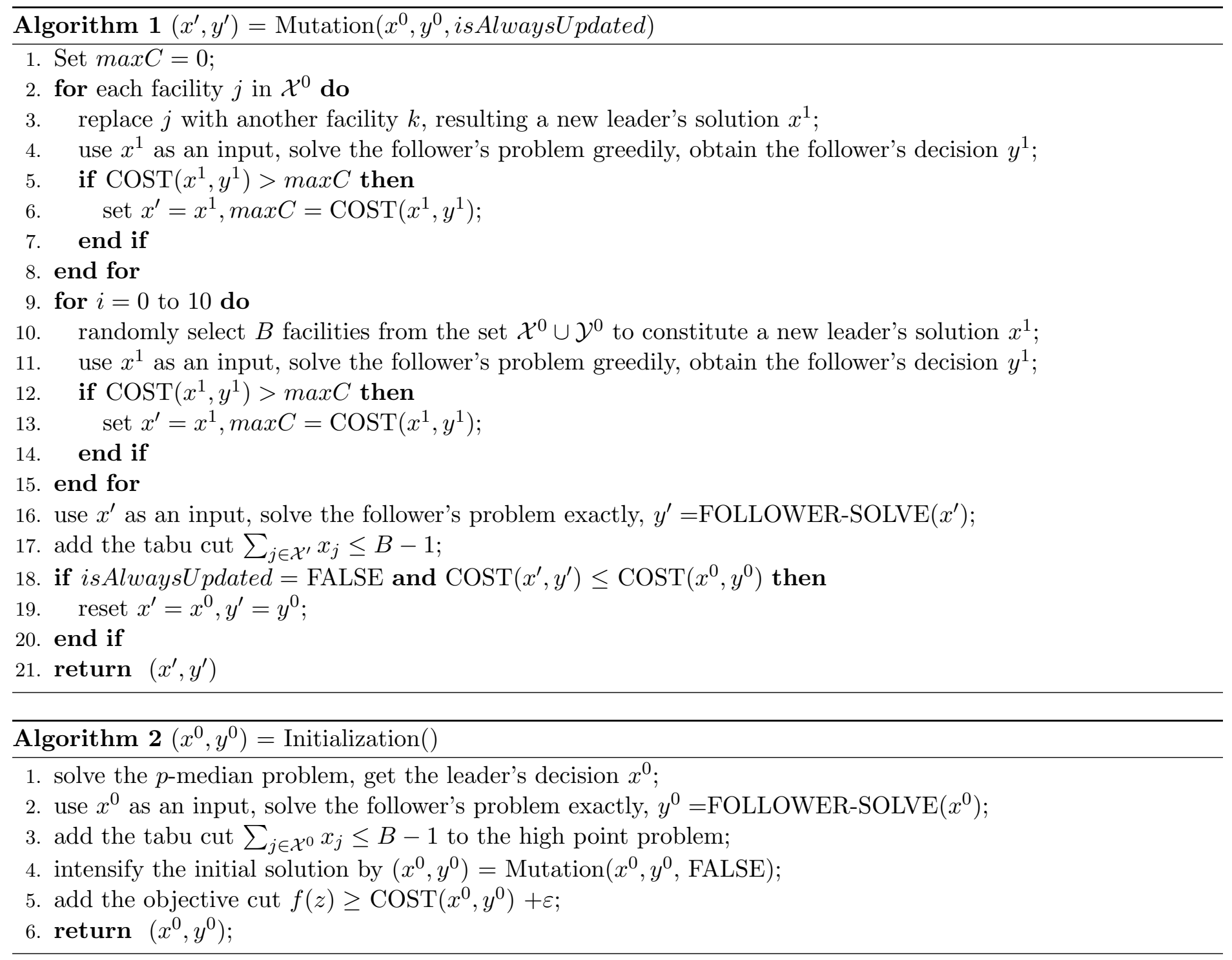

\subsection{Variable Fixing}

Danna et al. (2005) observe that in a general 0-1 MIP, some variables often take the same values in the optimal solution and in the LP relaxation solution. Therefore, if a variable has the same value in the incumbent and the LP relaxation solution, it is more likely that it will keep that value in the optimal solution. If such variables are fixed, then one can get a much smaller problem. Based on this observation, Danna et al. (2005) propose a relaxation induced neighborhood search (RINS) method. This variable fixing process is often called hard variable fixing (Bixby et al. 2000). We extend this idea in our VNDS heuristic.

At each iteration, we fix $B+K-l$ location variables to their values in the incumbent solution $\left(x^{0}, y^{0}\right)$, i.e., the subsequent local search (Section 4.5) will be performed on the subspace of $l$ variables. 
The decomposition procedure is presented in Algorithm 3. After this process, the number of free variables in the current problem is decreased.

Since the current leader's solution $x^{0}$ must have been tabu, the locations that are opened by the leader cannot all be fixed. In other words, at least one facility that belongs to the set $\mathcal{X}^{0}$ has to be released. So in the variable fixing process, we first randomly choose one facility $j$ from the leader's decision $\mathcal{X}^{0}$. Then the other $l-1$ facilities to be released are chosen from the set $\mathcal{X}^{0} \cup \mathcal{Y}^{0}$, according to their distance to the facility $j$. The remaining facilities will be fixed in the local search phase. This procedure is similar to the decomposition scheme used by Hansen et al. (2001) for the $p$-median problem.

Note that after fixing the location variables, the high point problem could be further reduced by fixing the assignment variables $w$ and $z$. For a problem with $R \geq 3$, suppose the facilities that are fixed by the leader and the follower are $\left\{j_{1}, j_{2}\right\}$ and $\left\{j_{3}, j_{4}, j_{5}\right\}$, respectively. For customer $i$, suppose the 3 closest facilities are $j_{1}, j_{2}$ and $j_{3}$. Then, as long as facilities $j_{1}, j_{2}$ and $j_{3}$ are open (and this is always the case, since they are fixed), customer $i$ will surely be assigned to $j_{1}$ at the first level, to $j_{2}$ at the second level and to $j_{3}$ at the third level. So we can fix $w_{i j_{1} 1}=1, w_{i j_{2} 2}=1, w_{i j_{3} 3}=1$ and $z_{i 1}=1, z_{i 2}=1, z_{i 3}=0$. Next, considering constraints (13), we can further set $w_{i j 1}=0, \forall j \in J \backslash\left\{j_{1}\right\}$, $w_{i j 2}=0, \forall j \in J \backslash\left\{j_{2}\right\}$ and $w_{i j 3}=0, \forall j \in J \backslash\left\{j_{3}\right\}$; regarding constraints (14), we can set $w_{i j_{1} r}=$ $0, \forall r \neq 1, w_{i j_{2} r}=0, \forall r \neq 2$ and $w_{i j_{3} r}=0, \forall r \neq 3$. Finally, if the number of follower's facilities that are fixed is equal to $K$, e.g., $K=3$ in this case, we can set $y_{j}=0, \forall j \in J \backslash\left\{j_{3}, j_{4}, j_{5}\right\}$.

After this variable fixing process, the scale of the problem will be decreased considerably, so that the solver will solve the reduced problem quite efficiently in the local search phase.

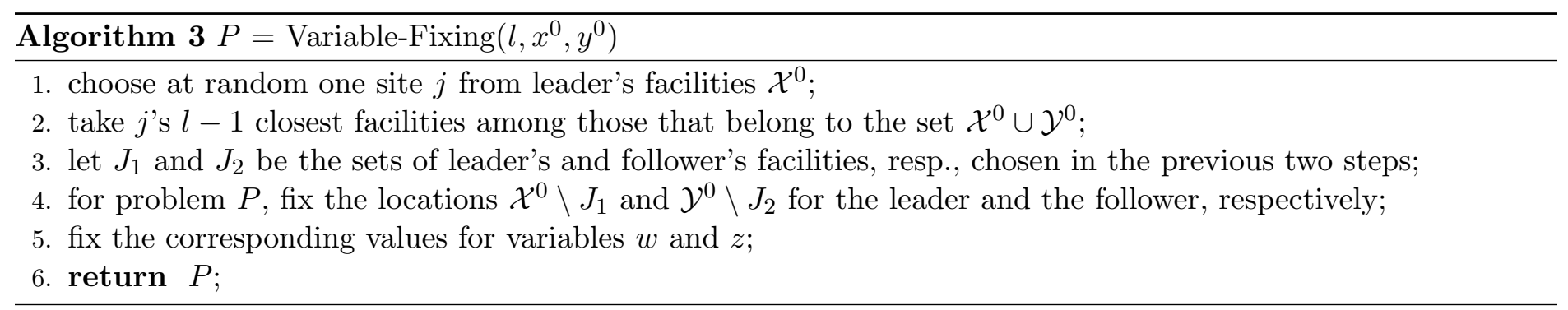

\subsection{Local Search}

Note that in our algorithm, we combine two approaches: hard variable fixing in the main scheme (Section 4.4) and soft variable fixing in the local search. Soft variable fixing is implemented by adding local branching cuts. The procedure of the local search phase is given in Algorithm 4. 
At each iteration of the Local Search procedure, a local branching cut $\Delta\left(x, x^{0}\right)+\Delta\left(y, y^{0}\right) \leq r h s$, with the incumbent solution $\left(x^{0}, y^{0}\right)$ and the current value of $r h s$, is added to the current problem (Step 3). Then the MIP solver is called to solve the high point problem (Step 4) within given node time limit $t_{m i p}$ and current best lower bound $\overline{L B}$. Thus, the search space for the solver is reduced, and a solution is expected to be found (or be proven infeasible) in a much shorter time than the time needed for the original problem without the local branching cut and without the variable fixing.

The subsequent steps depend on the status of the MIP solver. If the subproblem is solved to optimality (Step 6) or proved to be infeasible (including the case that the upper bound is lower than $\overline{L B}$ ) (Step 12 , we do not need to consider the current neighborhood in further solution space exploration, so the current local branching cut is reversed (Step 7 and Step 16). In case of infeasibility (Step 12), the neighborhood size is increased by one $(r h s=r h s+1$, Step 17); however, if $r h s \geq l$, the next solving must be infeasible so the local search terminates immediately (Step 14), since in the subproblem, the hard variable fixing requires that $B+K-l$ location variables are fixed to 1 , while the soft variable fixing imposes $B+K-r h s$ location variables to be 0 . If a feasible solution is found but has not been proven optimal (this new solution must be better than the incumbent $\left(x^{0}, y^{0}\right)$, since we update the objective cut once an improved lower bound is obtained), the last left branching is removed (Step 10), and later will be replaced by the new branching cut (Step 3). Finally, if the solver fails to find a feasible solution and also to prove the infeasibility of the current subproblem (Step 19), the local search phase is terminated.

We should emphasize that when the MIP solver is used to solve the problem, during the solving process, any time the solver finds an integer solution $\left(x^{1}, y^{1}\right)$, the follower problem is solved exactly to check whether $\left(x^{1}, y^{1}\right)$ is bilevel feasible or not. If it is bilevel feasible, now an improved solution is obtained; otherwise, if it is infeasible, we will get another follower's decision $\bar{y}^{1}$, and solution $\left(x^{1}, \bar{y}^{1}\right)$ is bilevel feasible. When the solver terminates, it will return a set of bilevel feasible solutions, consisting of the set $S$, along with a set of checked leader's solutions $x^{\prime}$ (Step 4). We can traverse the set $S$, and select the best one to update the incumbent solution $\left(x^{0}, y^{0}\right)$ (if the best one is better than the incumbent, Step 22). In addition, in order to avoid returning to the same solutions $\left(x^{\prime}\right)$ again during the search process, we need to add a tabu cut for each checked leader's solution (Step 25). Note that all branching cuts are temporary, they need to be removed at the end of the local search phase (Step 28), while all tabu cuts are static or permanent. 


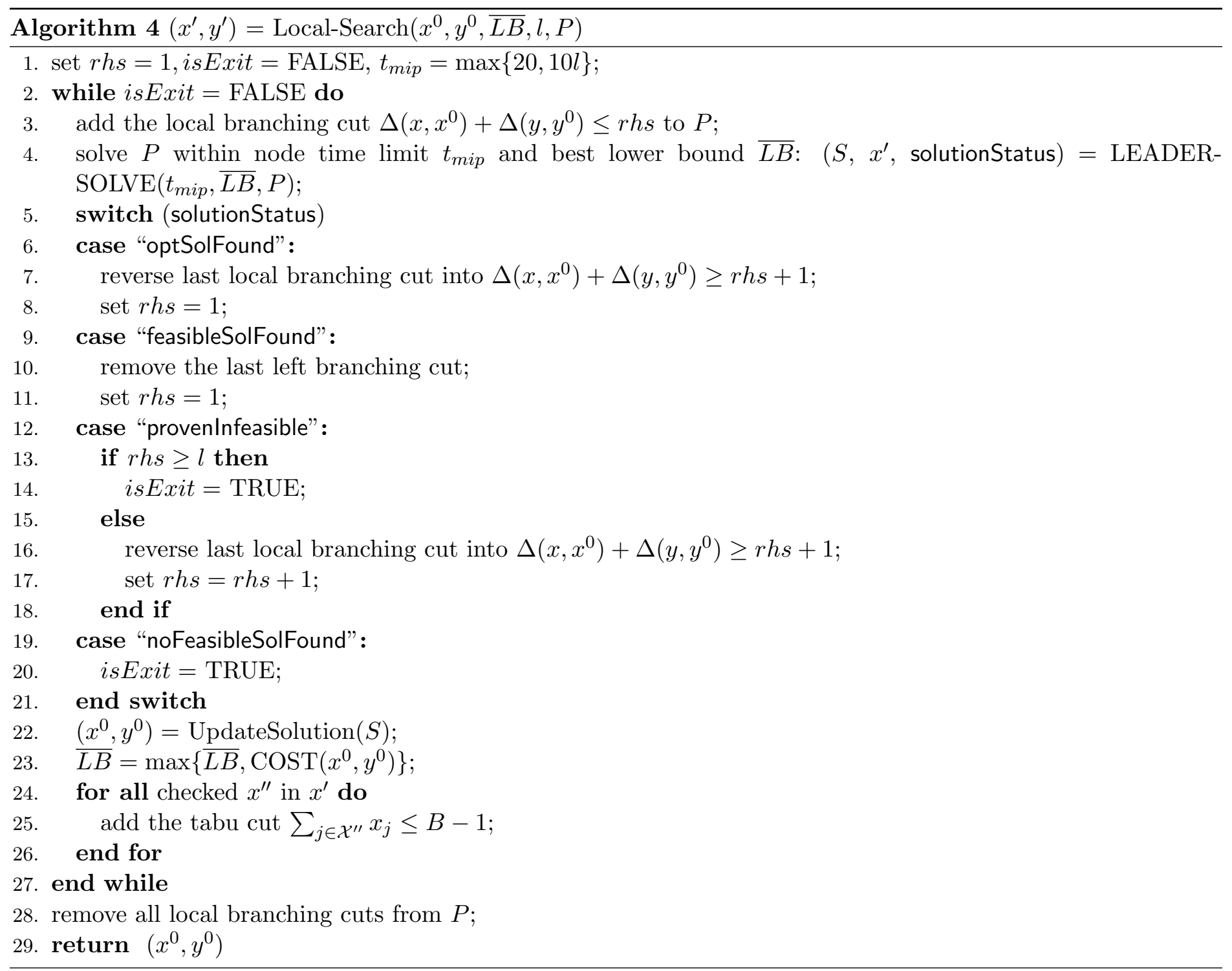

\subsection{The VNDS Algorithm}

Variable neighborhood search (VNS) (Hansen and Mladenović 2001, Hansen et al. 2010) is a recent metaheuristic that is based on the systematic change of neighborhoods, aiming for an ascent to local optimum and an escape from local valleys. Variable Neighborhood Search Branching is a heuristic for solving MIPs, using a general-purpose MIP solver as the black-box (Hansen et al. 2006). As in the local branching method, neighborhoods are defined by adding constraints to the original problem, thus reducing the solution space and yielding an easier problem. VNDS follows a general VNS scheme within a successive approximate decomposition method (Hansen et al.|2001). Readers may refer to these literatures for details. The main scheme of the proposed VNDS algorithm is presented in Algorithm 
5. consisting of all the sub-procedures introduced in the above sections. The basic idea is to define a variable fixing scheme for generating a sequence of smaller subproblems that are normally easier to solve than the original problem.

The local branching heuristic (Fischetti and Lodi 2003) and the general VNS scheme (Hansen et al. 2010) have many parameters. Here, we make our heuristic more user-friendly by reducing those parameters to only two, i.e., the outer iterations outerIter and the inner iterations innerIter in the following way: (i) The number of released variables $l$ satisfies $l \leq \min \{B+K$, innerIter $\}$ (Step 13 in Algorithm 5). If the value of $l$ exceeds this limit, we set $l=1$, i.e., we continue the decomposition by solving smaller subproblems. We observe that solutions can be improved quite fast in the early iterations of the algorithm even with small $l$. (ii) We allow an increase of the neighborhood size $r h s$ without limit (that is, we actually allow $r h s<l$ in Step 13 in Algorithm 4). (iii) We restrict the node time limit (in seconds) to solve the subproblem to be $t_{\text {mip }}=\max \{20,10 l\}$ for a given $l$ (Step 1 in Algorithm 4). (iv) We do not restrict the overall time to perform one local search phase, since in fact one phase can typically be completed quite quickly.

In Algorithm 5, at each iteration of the outer loop, the right-hand side of the objective cut should be set to the current best lower bound $\overline{L B}$ (Step 22). After the local search phase, if an improved solution is obtained, we will try to further intensify this solution (Step 7); while if parameter $l$ exceeds the maximal value allowed, we will diversify the best solution to find a new incumbent (Step 15).

Although Algorithm 5 is specifically designed to solve the CFLPD problem, it can be extended to other BBLPs, since it is based on mathematical optimization techniques (known as matheuristics) and the use of generic solvers. To solve general BBLPs, one only needs to reformulate the specific high point problem, and cut off bilevel infeasible solutions using valid inequality (24) through the callback interface of the MIP solver; the bounding, fathoming and branching procedures employed in a traditional LP-based branch-and-bound algorithm can be applied in a straightforward way. Then the checked solutions are visited from outside of the local search phase. For general problems, the hard variable fixing process is discussed by Danna et al. (2005), Lazić et al. (2010) and Hanafi et al. (2014), i.e., variables which have smaller distances between the incumbent and the LP relaxation values are fixed first. The other procedures remain the same. In Section 5.1, we test the performance of the proposed algorithm on the $(r \mid p)$-centroid problem. 


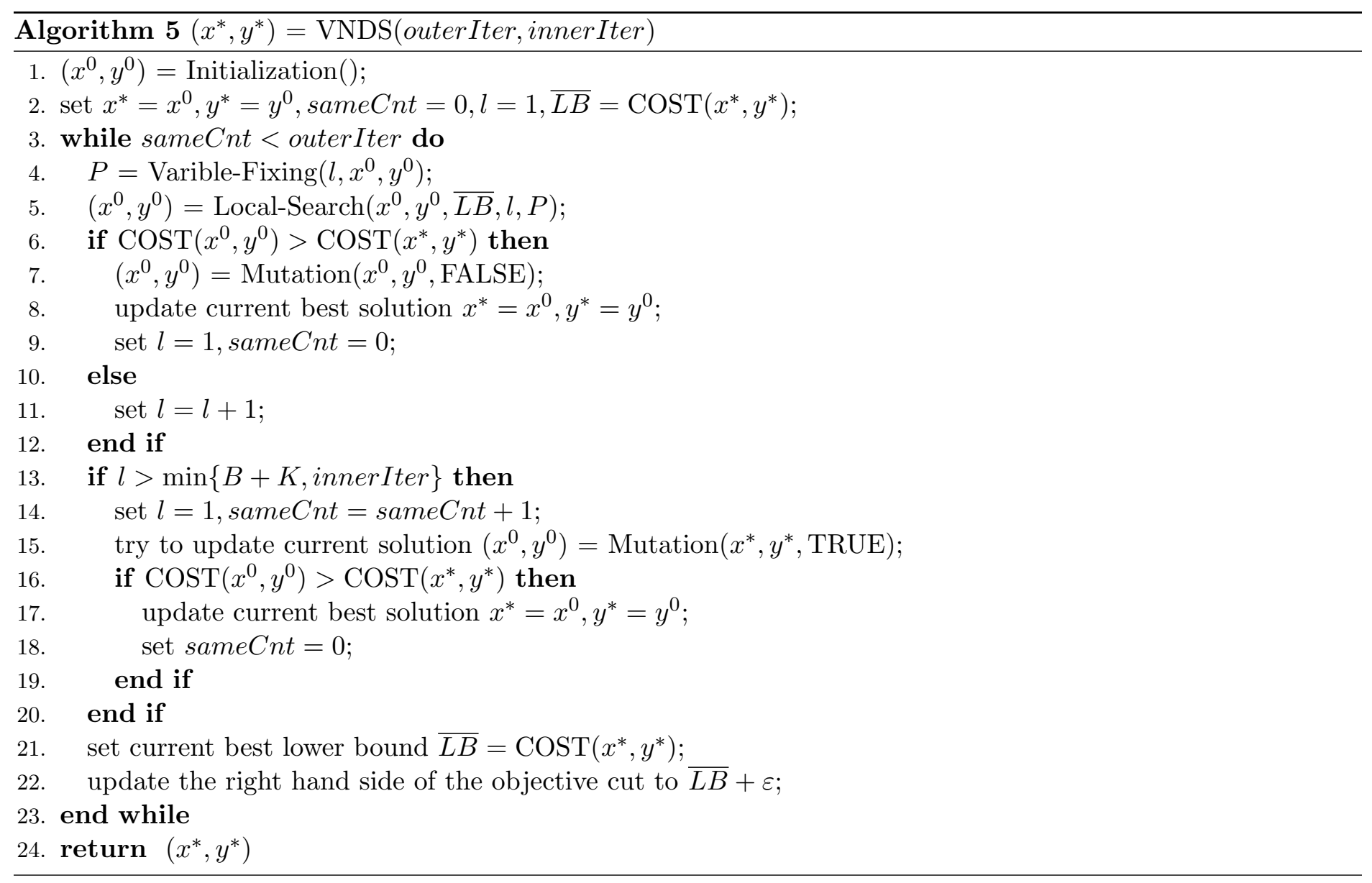

\section{$5 \quad$ Numerical Experiments}

In this section, we present computational results of proposed VNDS. All experiments are carried out on an Intel Core Dual PC, 2.53 GHz and 3GB RAM. We use Gurobi 6.0 as the optimization solver. In the algorithm, we simply set outerIter $=5$ and innerIter $=8$. (These two parameters can take larger values to explore a wider space, but we observed that this setting balances well between the scale and the desired quality of each subproblem solved.)

We test our method on two different group of instances. The first group is composed of 80 instances from the Discrete Location Problems benchmark library $5^{5}$ This dataset is to test the performance of the proposed VNDS on the RPCP compared with published best results. The second group is composed of extensive instances based on U.S. census data ${ }^{6}$ This dataset is to demonstrate the performance of proposed VNDS heuristic on the CFLPD problem, and to draw some managerial insights. In all tables that follow, the column labeled "TTBS" reports the "Time To Best Solution," i.e., the moment that

\footnotetext{
${ }^{5}$ The Discrete Location Problems dataset is available at http://math.nsc.ru/AP/benchmarks/english.html.

${ }^{6}$ The U.S. census dataset is available at http://coral.ie.lehigh.edu/ larry/research/publications/
} 
the best solution is obtained; and the "time" column reports the total computational time when the algorithm terminates; all computational times are measured in seconds.

Note that in all data sets, no two facility-customer pairs have the same distance; that is, all data sets satisfy the assumption made in Section 3.1.1.

\subsection{Computational Results for the RPCP}

In this section, we use the proposed VNDS heuristic to solve the RPCP (a special case of the CFLPD, as noted in $\S 1$ and compare its performance with that of the best available exact methods - MEM (Kochetov et al. 2013) and RP-B\&C (Roboredo and Pessoa|2013) — as well as the best available heuristic methods-VNS and STS (Davydov et al. 2014), TSL (Davydov 2012) and GA+solA (Biesinger et al. 2015).

Table 1: Comparison of the results from Euclidean class with $n=100, p=r=5, \mu_{i} \sim U(1,200)$

\begin{tabular}{|c|c|c|c|c|c|c|c|c|}
\hline \multirow[t]{2}{*}{ Inst } & \multirow[t]{2}{*}{$p$} & \multirow[t]{2}{*}{ optimal } & \multicolumn{2}{|c|}{ MEM } & \multicolumn{2}{|c|}{ RP-B\&C } & \multicolumn{2}{|c|}{ VNDS } \\
\hline & & & LB & time & LB & time & LB & TTBS \\
\hline 111 & 5 & 4139 & 4139 & 60 & 4139 & 1649.21 & 4139 & 53.66 \\
\hline 211 & 5 & 4822 & 4822 & 240 & 4822 & 9597.10 & 4822 & 62.13 \\
\hline 311 & 5 & 4215 & 4215 & 2280 & 4215 & 18823.93 & 4215 & 72.03 \\
\hline 411 & 5 & 4678 & 4678 & 4140 & 4678 & 4442.98 & 4678 & 6.12 \\
\hline 511 & 5 & 4599 & 4599 & 960 & 4599 & 28142.65 & 4599 & 25.89 \\
\hline 611 & 5 & 4483 & 4483 & 120 & 4483 & 1540.20 & 4483 & 70.12 \\
\hline 711 & 5 & 5153 & 5153 & 300 & 5153 & 7852.78 & 5153 & 18.56 \\
\hline 811 & 5 & 4404 & 4404 & 120 & 4404 & 11730.26 & 4404 & 62.50 \\
\hline 911 & 5 & 4700 & 4700 & 780 & 4700 & 17417.78 & 4700 & 22.12 \\
\hline 1011 & 5 & 4923 & 4923 & 60 & 4923 & 1100.63 & 4923 & 25.43 \\
\hline Avg. & - & 4611.6 & 4611.6 & 906 & 4611.6 & 10229.75 & 4611.6 & 41.86 \\
\hline
\end{tabular}

For all instances in the first group, customers and facilities are located at the same sites $(I=J)$, which are chosen randomly on a Euclidean plane of size $7000 \times 7000$. The distances $d_{i j}$ are the Euclidean distances between the sites $i$ and $j$. With respect to the customers' demand, there are two cases: $\mu_{i} \sim U(1,200), \forall i \in I$ and $\mu_{i}=1, \forall i \in I$. The number of sites is $n=100$, and the number of facilities to be opened is $p=r \in\{5,10,15,20,25,30\}$. (In the more general CFLPD, $p$ and $r$ are called $B$ and $K$, respectively.)

Tables 14 show experimental results for the proposed VNDS heuristic compared with the best published approaches. In particular, the first two tables report the case $\mu_{i} \sim U(1,200), \forall i \in I$, while the last two tables report the case $\mu_{i}=1, \forall i \in I$. In all tests, $p=r$, so we only show the value of $p$ in the tables. The GA+solA solves each instance 30 times, and the average of 30 runs are recorded in the tables, while the other methods have single runs for each instance. All heuristic methods record the 
Table 2: Comparison of the results from Euclidean class with $n=100, p=r \geq 10, \mu_{i} \sim U(1,200)$

\begin{tabular}{|c|c|c|c|c|c|c|c|c|c|c|c|c|c|c|}
\hline \multirow[t]{2}{*}{ Inst } & \multirow[t]{2}{*}{$p$} & \multirow[t]{2}{*}{ optimal } & \multicolumn{2}{|c|}{ STS } & \multicolumn{2}{|c|}{ TSL } & \multicolumn{2}{|c|}{ GA } & \multicolumn{2}{|c|}{ MEM } & \multicolumn{2}{|c|}{ RP-B\&C } & \multicolumn{2}{|c|}{ VNDS } \\
\hline & & & LB & TTBS & LB & TTBS & LB & TTBS & LB & time & LB & time & LB & TTBS \\
\hline 111 & 10 & 4361 & 4361 & 63.7 & 4361 & 342 & 4361 & 11.2 & 4361 & 3600 & 4361 & 10217 & 4361 & 27.8 \\
\hline 311 & 10 & 4483 & 4483 & 33.8 & 4483 & 819 & 4483 & 8.2 & 4483 & 8760 & 4483 & 19071.3 & 4483 & 89.1 \\
\hline 411 & 10 & 4994 & 4994 & 19.3 & 4994 & 270 & 4994 & 8.0 & 4994 & 1980 & 4994 & 13743.9 & 4994 & 50.2 \\
\hline 511 & 10 & 4906 & 4906 & 27.2 & 4906 & 396 & 4906 & 11.4 & 4906 & 23940 & 4906 & 80413.8 & 4906 & 102.4 \\
\hline 611 & 10 & 4595 & 4595 & 44.5 & 4595 & 504 & 4595 & 21.6 & 4595 & 8580 & 4595 & 51583.2 & 4595 & 90.6 \\
\hline 911 & 10 & 5302 & 5302 & 19.2 & 5302 & 297 & 5302 & 7.5 & 5302 & 360 & 5302 & 2377.9 & 5302 & 50.3 \\
\hline 1011 & 10 & 5005 & 5005 & 103.5 & 5005 & 333 & 5005 & 15.0 & 5005 & 5820 & 5005 & 33765.1 & 5005 & 21.2 \\
\hline Avg. & - & 4915.1 & 4915.1 & 52.4 & 4915.1 & 418 & 4915.1 & 11.9 & 4915.1 & 6906 & 4915.1 & 26782.2 & 4915.1 & 64.0 \\
\hline 111 & 15 & 4596 & 4596 & 173.3 & 4596 & 545 & 4596 & 16.3 & 4596 & 4320 & 4596 & 9752.0 & 4596 & 92.3 \\
\hline 211 & 15 & 5373 & 5373 & 88.9 & 5373 & 12021 & 5373 & 18.0 & 5373 & 230700 & 5373 & 80956.4 & 5373 & 46.1 \\
\hline 311 & 15 & 4800 & 4800 & 91.1 & 4800 & 3126 & 4800 & 18.9 & 4800 & 23700 & 4800 & 27707.3 & 4800 & 16.5 \\
\hline 811 & 15 & 4675 & 4675 & 123.4 & 4675 & 1088 & 4675 & 52.1 & 4675 & 274200 & 4675 & 115183.5 & 4675 & 62.3 \\
\hline 911 & 15 & 5158 & 5158 & 157.8 & 5158 & 1322 & 5158 & 240.1 & 5158 & $>36000$ & 5158 & $>36000$ & $\underline{5157}$ & 33.2 \\
\hline 1011 & 15 & 5195 & 5195 & 48.2 & 5195 & 2403 & 5195 & 28.2 & 5195 & $>36000$ & 5195 & 72034.4 & $\overline{5195}$ & 56.1 \\
\hline Avg. & - & 5070 & 5070 & 134.6 & 5069.2 & 2670 & 5069.2 & 53.7 & 5070 & $>102228$ & 5070 & $>58181$ & 5069.9 & 50.0 \\
\hline 111 & 20 & 4512 & 4484 & 118.1 & 4512 & 6008 & 4512 & 159.4 & 4512 & 60 & 4512 & $>36000$ & 4512 & 82.3 \\
\hline 211 & 20 & 5432 & 5432 & 289.2 & 5432 & 4922 & 5432 & 75.4 & 5432 & 11100 & 5432 & $>36000$ & 5432 & 142.1 \\
\hline 311 & 20 & 4893 & 4893 & 211.3 & 4893 & 9365 & 4893 & 85.4 & 4893 & 14880 & 4893 & $>36000$ & 4893 & 36.2 \\
\hline 411 & 20 & 5209 & 5209 & 288.9 & 5209 & 5165 & 5209 & 46.8 & 5209 & 300 & 5209 & $>36000$ & 5209 & 70.1 \\
\hline 511 & 20 & 5334 & 5334 & 133.2 & 5334 & 7922 & 5334 & 60.9 & 5334 & 6600 & 5334 & $>36000$ & 5334 & 82.1 \\
\hline 611 & 20 & 4952 & $\underline{4944}$ & 198.1 & 4952 & 13081 & 4952 & 58.1 & 4952 & 11400 & 4952 & $>36000$ & 4952 & 54.9 \\
\hline 711 & 20 & 5893 & 5893 & 254.3 & 5893 & 6000 & 5893 & 21.5 & 5893 & 5820 & 5893 & $>36000$ & 5893 & 44.2 \\
\hline 811 & 20 & 4858 & 4858 & 118.8 & 4858 & 8526 & 4858 & 22.9 & 4858 & 34200 & 4858 & $>36000$ & 4858 & 66.3 \\
\hline
\end{tabular}

"TTBS", i.e., the time needed for find the best solution; all exact methods record the total computation "time". The column "Inst" indicates the code name of the test instance; the column "LB" indicates the best lower bound found by each method. For each instance, the column "optimal" contains the leader's objective value for the optimal solution, if any. The "LB" found by each method is marked with an underline if it is strictly less than the optimal value. The results for all methods other than VNDS are taken directly from the sources cited above (not from our own independent tests). Not all papers report results for all sets of instances, so some of the Tables 14 contain more columns than others.

We observe that the proposed VNDS heuristic is able to find optimal solutions for 77 of the 80 instances, and the other 3 approximate solutions are very close to optimal. The performance of our heuristic is very similar to that of the best heuristic to date - the recent GA+solA heuristic (Biesinger et al. 2015) - and is very competitive in terms of CPU time with the other methods (exact and heuristic), 
Table 3: Comparison of the results from Euclidean class with $n=100, p=r=5$ and $10, \mu_{i}=1$

\begin{tabular}{|c|c|c|c|c|c|c|c|c|}
\hline \multirow[t]{2}{*}{ Inst } & \multirow[t]{2}{*}{$p$} & \multirow[t]{2}{*}{ optimal } & \multicolumn{2}{|c|}{ MEM } & \multicolumn{2}{|c|}{ RP-B\&C } & \multicolumn{2}{|c|}{ VNDS } \\
\hline & & & LB & $\mathrm{CPU}$ & LB & $\mathrm{CPU}$ & LB & TTBS \\
\hline 111 & 5 & 47 & 47 & 360 & 47 & 2653.16 & 47 & 24.10 \\
\hline 211 & 5 & 48 & 48 & 60 & 48 & 3523.85 & 48 & 38.12 \\
\hline 311 & 5 & 45 & 45 & 1560 & 45 & 12165.54 & 45 & 4.38 \\
\hline 411 & 5 & 47 & 47 & 120 & 47 & 3122.87 & 47 & 4.20 \\
\hline 511 & 5 & 47 & 47 & 60 & 47 & 2363.45 & 47 & 2.01 \\
\hline 611 & 5 & 47 & 47 & 180 & 47 & 3060.19 & 47 & 0.23 \\
\hline 711 & 5 & 47 & 47 & 240 & 47 & 3201.57 & 47 & 9.29 \\
\hline 811 & 5 & 48 & 48 & 60 & 48 & 2142.08 & 48 & 32.14 \\
\hline 911 & 5 & 47 & 47 & 120 & 47 & 2685.41 & 47 & 2.93 \\
\hline 1011 & 5 & 47 & 47 & 120 & 47 & 3973.72 & 47 & 7.49 \\
\hline Avg. & - & 47 & 47 & 288 & 47 & 3889.18 & 47 & 12.49 \\
\hline 111 & 10 & 50 & 50 & 780 & 50 & 2283.22 & 50 & 21.18 \\
\hline 211 & 10 & 49 & 49 & 1200 & 49 & 4707.05 & 49 & 8.12 \\
\hline 311 & 10 & 48 & 48 & 11700 & 48 & 13366.85 & 48 & 56.19 \\
\hline 411 & 10 & 49 & 49 & 8100 & 49 & 11318.52 & 49 & 28.10 \\
\hline 511 & 10 & 48 & 48 & 16200 & 48 & 18923.81 & 48 & 1.98 \\
\hline 611 & 10 & 47 & 47 & 54000 & 47 & 22906.99 & 47 & 45.19 \\
\hline 711 & 10 & 51 & 51 & 720 & 51 & 2530.35 & 51 & 34.72 \\
\hline 811 & 10 & 48 & 48 & 8700 & 48 & 15593.08 & $\underline{47}$ & 6.97 \\
\hline 911 & 10 & 49 & 49 & 6120 & 49 & 11238.96 & $\overline{49}$ & 14.02 \\
\hline 1011 & 10 & 49 & 49 & 10800 & 49 & 14228.92 & 49 & 38.01 \\
\hline Avg. & - & 48.8 & 48.8 & 11832 & 48.8 & 11709.78 & 48.7 & 25.45 \\
\hline
\end{tabular}

Table 4: Comparison of the results from Euclidean class with $n=100, p=r=25$ and $30, \mu_{i}=1$

\begin{tabular}{|c|c|c|c|c|c|c|c|}
\hline \multirow[t]{2}{*}{ Inst } & \multirow[t]{2}{*}{$p$} & \multicolumn{2}{|c|}{ VNS } & \multicolumn{2}{|c|}{ STS } & \multicolumn{2}{|c|}{ VNDS } \\
\hline & & LB & TTBS & LB & TTBS & $\mathrm{LB}$ & TTBS \\
\hline 111 & 25 & 53 & 33.14 & 53 & 56.88 & 53 & 82.13 \\
\hline 211 & 25 & 52 & 65.78 & 52 & 21.17 & 52 & 62.31 \\
\hline 311 & 25 & 57 & 80.48 & 57 & 12.16 & 57 & 55.19 \\
\hline 411 & 25 & 53 & 274.94 & 53 & 106.18 & 53 & 30.21 \\
\hline 511 & 25 & 53 & 18.84 & 53 & 37.14 & 53 & 102.87 \\
\hline 611 & 25 & 57 & 15.69 & 57 & 148.12 & $\underline{56}$ & 516.34 \\
\hline 711 & 25 & 54 & 18.71 & 54 & 8.14 & $\overline{54}$ & 332.09 \\
\hline 811 & 25 & 55 & 42.25 & 55 & 86.11 & 55 & 209.19 \\
\hline 911 & 25 & 58 & 46.18 & 58 & 89.17 & 58 & 18.02 \\
\hline 1011 & 25 & 55 & 39.65 & 55 & 64.81 & 55 & 385.28 \\
\hline Avg. & - & 54.7 & 63.57 & 54.7 & 62.99 & 54.6 & 179.36 \\
\hline 111 & 30 & 58 & 155.74 & 58 & 84.16 & 58 & 268.19 \\
\hline 211 & 30 & 56 & 58.35 & 56 & 119.57 & 56 & 276.12 \\
\hline 311 & 30 & 58 & 34.30 & 58 & 56.61 & 58 & 129.02 \\
\hline 411 & 30 & 55 & 64.56 & 55 & 33.98 & 55 & 302.19 \\
\hline 511 & 30 & 58 & 48.02 & 58 & 45.18 & 58 & 128.01 \\
\hline 611 & 30 & 59 & 25.67 & 59 & 27.11 & 59 & 441.01 \\
\hline 711 & 30 & 58 & 31.92 & 58 & 18.67 & 58 & 5.83 \\
\hline 811 & 30 & 57 & 40.52 & 57 & 25.94 & 57 & 6.98 \\
\hline 911 & 30 & 59 & 15.71 & 59 & 35.85 & 59 & 11.92 \\
\hline 1011 & 30 & 59 & 18.24 & 59 & 67.94 & 59 & 10.92 \\
\hline Avg. & - & 57.7 & 49.30 & 57.7 & 51.50 & 57.7 & 158.02 \\
\hline
\end{tabular}


though of course a head-to-head comparison of CPU times is not possible due to differences in the platforms and environments in which the various methods were implemented and tested. The exact approaches can guarantee optimality but are very computationally intensive.

The results shown in Table 4 are to study the algorithm's behavior on the hardest values of parameters $p$ and $r$, i.e., $p=r=25,30$. Previously, only Davydov et al. (2014) have reported computational experiments for such values, so we compare with them. In these cases, exact methods are already unable to find a solution for the problem. Table 4 indicates that the proposed VNDS heuristic is able to find 19 of the same solutions as VNS and STS out of 20 tests. It has been observed by Alekseeva et al. (2010) that under fixed values of $|I|$ and $|J|$, the problem becomes more difficult when $p$ and $r$ are greater than or equal to about a one-third of $|J|$. In this case, considerable computational time is needed to solve the follower's problem and check bilevel feasibility.

It is not an easy task to establish a fair comparison of the efficiency of various algorithms, since they are running on different hardware platforms and use different MIP solvers. Nevertheless, our method is the first attempt to use a general MIP matheuristic to this very challenging computational setting. Based on the analysis above, we can conclude that VNDS is a good method for approximately solving RPCP since it is able to tackle instances with up to 100 customers, 100 potential facilities and $p=r=30$ in a reasonable time. In the next section, we are interested in its performance on the CFLPD.

\subsection{Computational Results for the CFLPD}

In this section, we test the performance of the VNDS heuristic using the second group of instances. Numerical tests are performed with different parameter settings on both the 49-node dataset and the 88-node dataset. Results are shown in Tables 58 and Figures 13.

\subsubsection{Performance of the VNDS method}

First, we design two enumeration methods to verify the performance of the proposed VNDS heuristic based on the 49-node dataset. Comparisons are shown in Table 5, in which $|J|=20$ means that we take the first 20 cities from the 49 cities in the dataset as the potential facilities (while $I$ contains all 49 cities). In each test, the leader has $\left(\begin{array}{c}B \\ |J|\end{array}\right)$ number of choices; while for a given leader's decision, the follower will react in the way that captures the most customers. If the follower's decision is enumerated

among all the $\left(\begin{array}{c}K \\ |J|-B\end{array}\right)$ choices, this method is named as Enum1; if the follower's decision is determined by solving the follower's problem (7)-15) exactly, this method is named as Enum2. Once both players 
Table 5: Comparison with the enumeration method

\begin{tabular}{|c|c|c|c|c|c|c|c|c|c|c|c|c|c|c|}
\hline \multirow[t]{2}{*}{ No. } & \multirow[t]{2}{*}{$|J|$} & \multirow[t]{2}{*}{$B$} & \multirow[t]{2}{*}{$K$} & \multirow[t]{2}{*}{$q$} & \multirow[t]{2}{*}{$R$} & \multirow[t]{2}{*}{$\left(\begin{array}{c}B \\
|J|\end{array}\right)$} & \multirow[t]{2}{*}{$\left(\begin{array}{c}K \\
|J|-B\end{array}\right)$} & \multirow[t]{2}{*}{ optimal } & \multirow{2}{*}{$\begin{array}{r}\text { Enum1 } \\
\text { time }\end{array}$} & \multirow{2}{*}{$\frac{\text { Enum2 }}{\text { time }}$} & \multicolumn{4}{|c|}{ VNDS } \\
\hline & & & & & & & & & & & \#update & \#checked & TTBS & time \\
\hline 1 & 15 & 4 & 4 & 0.10 & 1 & 1365 & 330 & 1137.4 & 20.4 & 17.6 & 2 & 36 & 0.4 & 2.4 \\
\hline 2 & 15 & 4 & 4 & 0.10 & 2 & 1365 & 330 & 1267.1 & 21.3 & 89.4 & 1 & 18 & 0.2 & 7.5 \\
\hline 3 & 15 & 4 & 4 & 0.10 & 3 & 1365 & 330 & 1276.6 & 22.3 & 280.6 & 1 & 13 & 0.6 & 13.2 \\
\hline 4 & 15 & 4 & 4 & 0.10 & 4 & 1365 & 330 & 1277.9 & 24.7 & 492.6 & 1 & 18 & 1.0 & 24.5 \\
\hline 5 & 16 & 2 & 2 & 0.10 & 3 & 120 & 91 & 1225.3 & 0.4 & 74.6 & 2 & 17 & 5.3 & 18.9 \\
\hline 6 & 16 & 4 & 2 & 0.10 & 3 & 1820 & 66 & 1723.8 & 5.2 & 433.8 & 1 & 21 & 0.5 & 27.6 \\
\hline 7 & 16 & 6 & 2 & 0.10 & 3 & 8008 & 45 & 1972.4 & 18.2 & 1059.9 & 2 & 28 & 1.1 & 33.2 \\
\hline 8 & 16 & 8 & 2 & 0.10 & 3 & 12870 & 28 & 2176.1 & 36.9 & 803.8 & 2 & 21 & 0.2 & 10.6 \\
\hline 9 & 16 & 2 & 4 & 0.10 & 3 & 120 & 1001 & 702.4 & 4.7 & 70.1 & 2 & 25 & 4.8 & 21.6 \\
\hline 10 & 16 & 2 & 6 & 0.10 & 3 & 120 & 3003 & 680.2 & 20.0 & 61.1 & 2 & 18 & 6.4 & 34.0 \\
\hline 11 & 16 & 2 & 8 & 0.10 & 3 & 120 & 3003 & 680.2 & 26.6 & 46.8 & 1 & 12 & 0.9 & 12.1 \\
\hline 12 & 20 & 3 & 3 & 0.10 & 3 & 1140 & 680 & 1212.8 & 29.6 & 899.1 & 2 & 24 & 6.0 & 59.4 \\
\hline 13 & 30 & 3 & 3 & 0.10 & 3 & 4060 & 2925 & 1180.8 & 449.6 & 6441.4 & 2 & 73 & 22.6 & 127.8 \\
\hline 14 & 40 & 3 & 3 & 0.10 & 3 & 9880 & 7770 & 1172.1 & 2907.6 & 24409.1 & 2 & 77 & 53.2 & 148.5 \\
\hline 15 & 20 & 5 & 5 & 0.02 & 3 & 15504 & 3003 & 1388.5 & 3110.4 & 4503.7 & 3 & 58 & 63.4 & 139.3 \\
\hline 16 & 20 & 5 & 5 & 0.04 & 3 & 15504 & 3003 & 1381.9 & 3088.3 & 3871.0 & 2 & 62 & 29.4 & 137.1 \\
\hline 17 & 20 & 5 & 5 & 0.06 & 3 & 15504 & 3003 & 1375.6 & 3102.0 & 3747.5 & 3 & 49 & 61.6 & 99.2 \\
\hline 18 & 20 & 5 & 5 & 0.08 & 3 & 15504 & 3003 & 1369.5 & 3093.8 & 3811.3 & 4 & 83 & 81.8 & 181.0 \\
\hline 19 & 20 & 5 & 5 & 0.10 & 3 & 15504 & 3003 & 1363.6 & 3109.1 & 3916.5 & 3 & 67 & 56.3 & 144.1 \\
\hline 20 & 20 & 5 & 5 & 0.20 & 3 & 15504 & 3003 & 1331.2 & 3082.7 & 4432.9 & 2 & 16 & 2.5 & 21.4 \\
\hline Avg. & - & - & - & - & - & 6837 & 1898 & 1294.8 & 1108.7 & 2973.1 & 2.0 & 36.8 & 19.9 & 63.2 \\
\hline
\end{tabular}

have made their decisions, customers are assigned to their facilities level by level in increasing order of distance. Both enumeration methods find the optimal solution; the only difference is their computation times. The last four columns report the results of the VNDS heuristic. Once the algorithm terminates, column "\#update" shows the number of updates of the best solution, column "\#checked" shows the total number of bilevel feasible solutions found.

We use Enum1 and Enum2 to solve the 20 tests in Table 5 to optimality. The VNDS heuristic can find all the optimal solutions, since the best LB found is equal to the optimal solution upon termination (so we only show the optimal one in the ninth column). Enum1 is quite efficient for very small instances, i.e., $\# 5$, \#6 and \#9, since the number of feasible solutions, $\left(\begin{array}{c}B \\ |J|\end{array}\right)\left(\begin{array}{c}K \\ |J|-B\end{array}\right)$, is not too large. Enum2 is a bit slower in general. This is because for small sized instances, enumerating the follower's decision is more efficient than solving the follower's problem directly. The proposed VNDS method has the best performance among the three methods in terms of CPU time (except for some very small instances). The best LB can be found even during the initialization process (Step 4 of Algorithm 2), which is shown in the column "\#update". The total number of checked solutions is also very small, as shown in the column "\#checked", indicating the intelligent direction guided by the local search mechanism. 
Table 5 shows that the proposed VNDS method performs well on the CFLPD. Next, we use it to solve larger instances. Tables 6 and 7 show the results for the 49 -node dataset. In these two tables, the "LB" column means the best lower bound found; the "share" column represents the leader's market share (in \%) in the best solution obtained; the last two columns, for each test, show the facilities that are opened by the leader and the follower, respectively. Furthermore, we select two parameters from $B, K, q, R$ and $|J|$, while keeping the other parameters unchanged, then show how these two parameters will affect the leader's market share; these results are illustrated in Figures 1, 2 and 3(a). The tests in Figure 3(a) and Figure 3(b) have the same parameter settings, except that Figure 3(b) shows the changes in the leader's objective value.

Table 6: Computational results on the CFLPD (49-node case, $|I|=49,|J|=20,30,40$ )

\begin{tabular}{|c|c|c|c|c|c|c|c|c|c|c|c|}
\hline No. & $|J|$ & $B$ & $K$ & $q$ & $R$ & LB & share & TTBS & time & Leader & Follower \\
\hline 1 & 20 & 5 & 5 & 0.02 & 3 & 1388.49 & 56.20 & 63.36 & 139.31 & $1,2,7,11,15$ & $3,4,5,6,18$ \\
\hline 2 & 20 & 5 & 5 & 0.04 & 3 & 1381.87 & 55.94 & 29.37 & 137.05 & $1,2,7,11,15$ & $3,4,5,6,18$ \\
\hline 3 & 20 & 5 & 5 & 0.06 & 3 & 1375.57 & 55.69 & 61.55 & 99.24 & $1,2,7,11,15$ & $3,4,5,6,18$ \\
\hline 4 & 20 & 5 & 5 & 0.08 & 3 & 1369.52 & 55.46 & 81.75 & 180.97 & $1,2,7,11,15$ & $3,4,5,6,18$ \\
\hline 5 & 20 & 5 & 5 & 0.10 & 3 & 1363.64 & 55.25 & 56.34 & 144.10 & $1,2,7,11,15$ & $3,4,5,6,18$ \\
\hline 6 & 20 & 5 & 5 & 0.20 & 3 & 1331.24 & 54.32 & 2.51 & 21.42 & $1,2,7,11,15$ & $3,4,6,14,19$ \\
\hline 7 & 30 & 10 & 10 & 0.10 & 1 & 1346.61 & 60.09 & 45.93 & 76.79 & $1,2,3,5,6,7,12,15,22,23$ & $4,8,9,10,11,20,21,24,27,29$ \\
\hline 8 & 30 & 10 & 10 & 0.10 & 2 & 1472.47 & 60.20 & 94.74 & 159.79 & $1,2,3,5,6,7,12,15,22,23$ & $9,10,11,18,21,26,27,30$ \\
\hline 9 & 30 & 10 & 10 & 0.10 & 3 & 1481.32 & 60.02 & 87.03 & 261.26 & $1,2,3,5,6,7,12,15,22,23$ & $9,10,11,18,21,26,27,30$ \\
\hline 10 & 30 & 10 & 10 & 0.10 & 4 & 1482.76 & 60.02 & 27.47 & 207.48 & $1,2,3,5,6,7,12,15,22,23$ & $4,8,9,10,11,21,26,27,29,30$ \\
\hline 11 & 30 & 10 & 10 & 0.10 & 5 & 1482.87 & 60.02 & 228.86 & 446.02 & $1,2,3,5,6,7,12,15,22,23$ & $4,8,9,10,11,18,21,26,27,30$ \\
\hline 12 & 40 & 2 & 2 & 0.10 & 3 & 1123.14 & 45.51 & 81.49 & 251.86 & 34,36 & 12,23 \\
\hline 13 & 40 & 4 & 4 & 0.10 & 3 & 1172.38 & 47.50 & 192.06 & 432.15 & $1,9,23,32$ & $2,14,19,31$ \\
\hline 14 & 40 & 6 & 6 & 0.10 & 3 & 1281.91 & 51.94 & 241.79 & 435.81 & $1,2,5,6,22,28$ & $3,4,14,19,36,39$ \\
\hline 15 & 40 & 8 & 8 & 0.10 & 3 & 1387.93 & 56.24 & 218.77 & 451.10 & $1,2,3,5,6,7,12,22$ & $4,9,10,20,23,27,33,39$ \\
\hline 16 & 40 & 10 & 10 & 0.10 & 3 & 1412.89 & 57.25 & 32.79 & 319.41 & $1,2,3,5,6,7,12,22,23,36$ & $4,8,9,10,11,14,20,27,33,39$ \\
\hline Avg. & - & - & - & - & - & 1365.91 & 55.73 & 96.61 & 235.23 & & 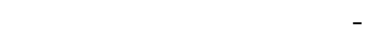 \\
\hline
\end{tabular}

The proposed VNDS approach uses the heuristic described in Algorithm 2 to find an initial solution to provide a lower bound of good quality. In fact, high-quality solutions can be found by VNDS after only a few rounds of fixing when driven by the local branching paradigm. Tables 6 and 7 show that good solutions can be found in an average of 4 minutes. As the value of $\left(\begin{array}{c}K \\ |J|-B\end{array}\right)$ increases, the time to solve the follower's problem exactly increases, so the computational time increases significantly. Thus, for more difficult instances, it will be important to develop exact approaches to efficiently solve the follower's problem.

In what follows, we will explore the effects of the parameters $B, K, q, R$ and $|J|$ on the optimal facility locations, and study the market share. 
Table 7: Computational results on the CFLPD (49-node case, $|I|=|J|=49$ )

\begin{tabular}{rrrrrrrrrrrr}
\hline No. & $|J|$ & $B$ & $K$ & $q$ & $R$ & LB & share & TTBS & time & Leader & Follower \\
\hline 1 & 49 & 5 & 3 & 0.10 & 2 & 1554.70 & 63.57 & 359.20 & 571.93 & $1,9,11,14,32$ & $37,40,47$ \\
2 & 49 & 5 & 4 & 0.10 & 2 & 1351.11 & 55.24 & 138.18 & 431.41 & $1,2,14,22,28$ & $15,23,39,47$ \\
3 & 49 & 5 & 5 & 0.10 & 2 & 1207.16 & 49.36 & 84.47 & 401.85 & $1,2,14,22,28$ & $4,7,15,39,47$ \\
4 & 49 & 5 & 6 & 0.10 & 2 & 1082.10 & 44.24 & 122.90 & 473.37 & $1,2,6,7,22$ & $4,15,23,27,39,47$ \\
5 & 49 & 5 & 7 & 0.10 & 2 & 1001.92 & 40.96 & 39.26 & 419.95 & $1,2,6,7,22$ & $4,14,15,19,20,27,39$ \\
6 & 49 & 3 & 5 & 0.05 & 2 & 706.68 & 28.68 & 199.00 & 419.59 & $1,2,6$ & $12,15,23,27,39$ \\
7 & 49 & 4 & 5 & 0.05 & 2 & 995.71 & 40.40 & 64.16 & 370.94 & $1,2,6,22$ & $12,15,23,27,39$ \\
8 & 49 & 5 & 5 & 0.05 & 2 & 1216.88 & 49.38 & 57.87 & 356.43 & $1,2,14,22,28$ & $4,7,15,27,39$ \\
9 & 49 & 6 & 5 & 0.05 & 2 & 1422.81 & 57.74 & 29.73 & 271.41 & $1,2,6,7,22,28$ & $3,4,15,19,39$ \\
10 & 49 & 7 & 5 & 0.05 & 2 & 1593.97 & 64.68 & 3.98 & 195.88 & $1,2,6,7,19,22,28$ & $3,4,5,15,39$ \\
11 & 49 & 4 & 4 & 0.02 & 3 & 1145.29 & 46.36 & 176.03 & 427.86 & $1,2,6,22$ & $4,14,15,27$ \\
12 & 49 & 4 & 4 & 0.04 & 3 & 1147.13 & 46.44 & 1068.65 & 1180.38 & $1,2,6,22$ & $4,14,15,27$ \\
13 & 49 & 4 & 4 & 0.06 & 3 & 1149.65 & 46.37 & 49.45 & 524.45 & $1,9,23,32$ & $14,27,31,47$ \\
14 & 49 & 4 & 4 & 0.08 & 3 & 1161.32 & 47.03 & 374.67 & 887.55 & $1,9,23,32$ & $14,27,31,47$ \\
15 & 49 & 4 & 4 & 0.10 & 3 & 1171.88 & 47.48 & 240.07 & 910.48 & $1,9,23,32$ & $14,27,31,47$ \\
16 & 49 & 4 & 4 & 0.20 & 3 & 1199.89 & 48.96 & 2716.07 & 3550.06 & $1,9,23,32$ & $11,14,27,37$ \\
17 & 49 & 4 & 4 & 0.05 & 1 & 1085.94 & 46.27 & 12.62 & 261.07 & $1,2,6,22$ & $4,14,15,27$ \\
18 & 49 & 4 & 4 & 0.05 & 2 & 1144.80 & 46.45 & 42.42 & 424.91 & $1,5,14,31$ & $6,9,23,33$ \\
19 & 49 & 4 & 4 & 0.05 & 3 & 1146.46 & 46.41 & 552.16 & 839.40 & $1,2,23,28$ & $14,17,39,47$ \\
20 & 49 & 4 & 4 & 0.05 & 4 & 1146.51 & 46.41 & 625.27 & 1069.01 & $1,2,23,28$ & $5,14,17,39$ \\
21 & 49 & 4 & 4 & 0.05 & 5 & 1146.51 & 46.39 & 171.75 & 747.84 & $1,2,23,28$ & $5,14,17,39$ \\
\hline Avg. & - & - & - & - & - & 1179.92 & 48.04 & 339.42 & 701.70 & & - \\
\hline
\end{tabular}

\subsubsection{Number of Located Facilities $B$ and $K$}

To investigate the influence of the parameters $B$ and $K$, we first assume that $B=K$. In either Figure 1 or Figure 3(a), the leader's market share increases when $B$ and $K$ grow. The leader obtains half of the market for $B=K=5$. The case $B=K=4.4$ is a demarcation point: If larger than that, the leader will dominate the market; if smaller than that, the follower will dominate the market. Based on the assumption that two players cannot operate at the same site, a large $B$ favors the leader because the leader can choose all the "good" locations first, and then dominates the market via these good locations. This is the first-mover advantage. This conclusion is also demonstrated in tests \#12-\#16 from Table 6 .

Then, we assume that $B \neq K$ to study how the two players' decisions influence each other. From tests \#1-\#10 in Table 7, we observe that the leader's market share decreases when $K$ increases and vice versa. If the players open different numbers of facilities, then the one that opens more will attract more market share. In contrast, we assume that both players open the same number of facilities, and this number is a constant. For \#1-\#11 in Table 6 and \#11-\#21 in Table 7, we observe that the facilities that the leader opens are basically the same, no matter how the other parameters change. However, for the same leader's solution, the follower may react with different responses (e.g., \#7 and 


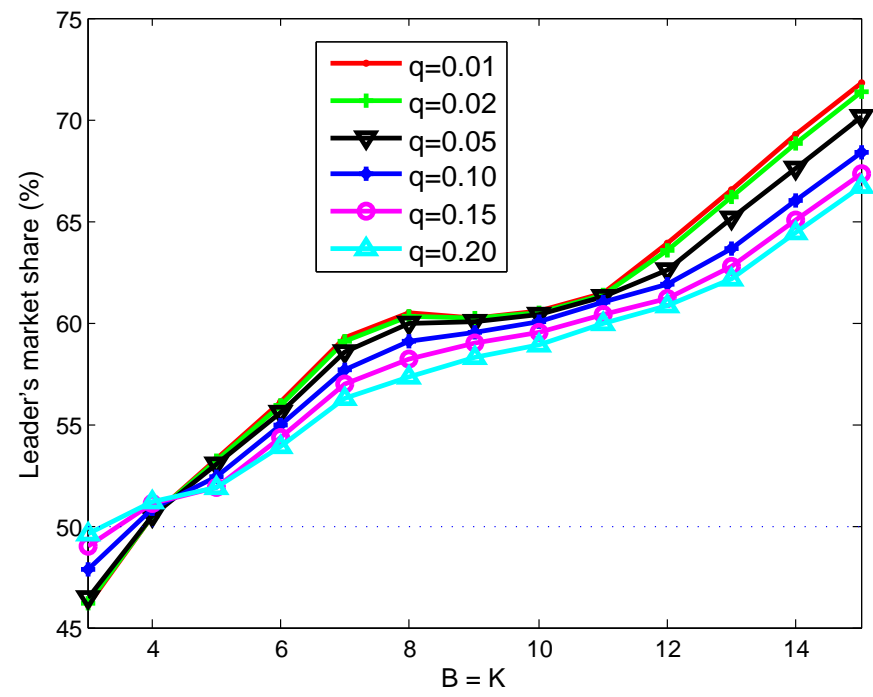

Figure 1: $|J|=30, R=3$

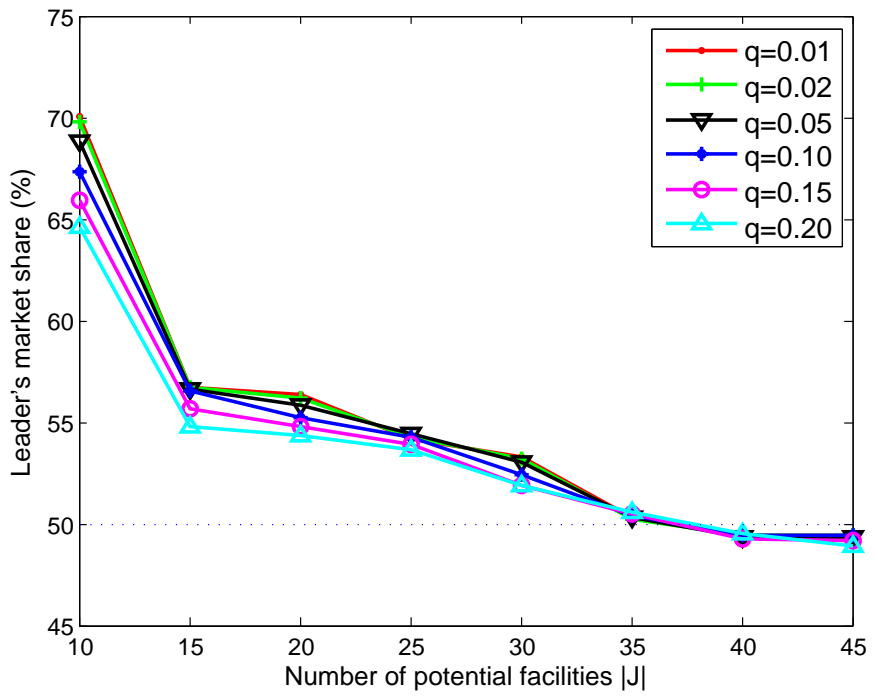

Figure 2: $B=K=5, R=3$

Table 8: Computational results on the CFLPD (88-node case, $|I|=88$ )

\begin{tabular}{|c|c|c|c|c|c|c|c|c|c|c|c|}
\hline No. & $|J|$ & $B$ & $K$ & $q$ & $R$ & LB & share & TTBS & time & Leader & Follower \\
\hline 2 & 30 & 5 & 5 & 0.02 & 3 & 2656.96 & 59.25 & 21.42 & 229.88 & $1,2,3,18,27$ & $5,6,8,14,23$ \\
\hline 3 & 30 & 5 & 5 & 0.05 & 3 & 2631.33 & 58.69 & 203.38 & 413.05 & $1,2,3,18,27$ & $5,6,8,13,14$ \\
\hline 5 & 30 & 5 & 5 & 0.20 & 3 & 2480.72 & 55.77 & 58.73 & 439.41 & $1,2,3,8,19$ & $5,14,16,18,28$ \\
\hline 6 & 40 & 10 & 10 & 0.05 & 1 & 2681.14 & 62.94 & 32.24 & 357.79 & $1,2,3,4,8,19,23,33,36,39$ & $5,7,9,11,14,17,18,27,29,32$ \\
\hline 7 & 40 & 10 & 10 & 0.05 & 2 & 2757.74 & 61.66 & 38.64 & 371.98 & $1,2,3,4,8,19,23,36,38,39$ & $5,6,7,11,13,14,17,18,27,29$ \\
\hline 10 & 40 & 10 & 10 & 0.05 & 5 & 2763.93 & 61.64 & 306.52 & 740.60 & $1,2,3,4,8,19,23,33,36,39$ & $5,7,9,11,14,17,18,27,29,32$ \\
\hline 11 & 50 & 2 & 2 & 0.02 & 3 & 2135.96 & 47.63 & 238.33 & 789.96 & 19,29 & 12,26 \\
\hline 12 & 50 & 3 & 3 & 0.02 & 3 & 2329.09 & 51.94 & 347.82 & 880.05 & $1,2,3$ & $5,13,46$ \\
\hline 13 & 50 & 4 & 4 & 0.02 & 3 & 2511.32 & 56.01 & 230.43 & 531.86 & $1,2,3,8$ & $4,5,44,46$ \\
\hline 14 & 50 & 5 & 5 & 0.02 & 3 & 2541.31 & 56.67 & 124.42 & 603.75 & $1,2,3,18,27$ & $5,8,32,44,46$ \\
\hline 15 & 50 & 6 & 6 & 0.02 & 3 & 2603.80 & 58.07 & 101.32 & 595.68 & $1,2,3,18,19,27$ & $4,8,23,32,37,46$ \\
\hline 16 & 40 & 3 & 3 & 0.05 & 2 & 2333.43 & 52.17 & 87.70 & 279.70 & $1,2,3$ & $5,13,32$ \\
\hline 21 & 88 & 10 & 10 & 0.01 & 3 & 2702.37 & 60.27 & 288.11 & 711.40 & $1,2,3,7,9,27,39,50,59,82$ & $4,5,8,14,23,32,36,38,58,80$ \\
\hline 22 & 88 & 10 & 10 & 0.02 & 3 & 2693.75 & 60.07 & 922.28 & 1229.15 & $1,2,3,7,9,27,39,50,59,82$ & $4,8,14,23,32,36,38,58,72,80$ \\
\hline 23 & 88 & 10 & 10 & 0.05 & 3 & 2669.22 & 59.53 & 1057.83 & 1692.84 & $1,2,3,7,9,27,39,50,59,82$ & $4,8,14,23,32,36,38,58,72,80$ \\
\hline 24 & 88 & 10 & 10 & 0.10 & 3 & 2652.92 & 59.22 & 2760.29 & 3767.48 & $1,2,3,5,7,27,33,39,59,67$ & $4,8,13,15,23,32,41,47,55,83$ \\
\hline 25 & 88 & 10 & 10 & 0.20 & 3 & 2595.24 & 58.34 & 3154.47 & 4704.79 & $1,2,3,5,6,7,27,36,39,50$ & $4,8,9,12,14,32,58,72,80,84$ \\
\hline 26 & 88 & 8 & 8 & 0.05 & 3 & 2627.40 & 58.60 & 4041.99 & 5269.69 & $1,2,3,7,27,50,59,82$ & $4,8,23,32,36,41,47,72$ \\
\hline 27 & 88 & 9 & 9 & 0.05 & 3 & 2672.84 & 59.62 & 1049.10 & 2638.15 & $1,2,3,7,27,39,50,59,82$ & $4,5,14,23,32,36,38,58,80$ \\
\hline 28 & 88 & 10 & 10 & 0.05 & 3 & 2669.22 & 59.53 & 1082.72 & 2106.64 & $1,2,3,7,9,27,39,50,59,82$ & $4,8,14,23,32,36,38,58,72,80$ \\
\hline 29 & 88 & 11 & 11 & 0.05 & 3 & 2676.55 & 59.70 & 702.98 & 2588.02 & $1,2,3,4,7,8,33,36,39,67,82$ & $5,9,11,14,18,23,27,32,50,58,71$ \\
\hline 30 & 88 & 12 & 12 & 0.05 & 3 & 2726.64 & 60.81 & 1456.75 & 2798.57 & $1,2,3,4,5,7,8,38,39,44,67,71$ & $6,11,13,14,23,27,36,50,57,66,70,83$ \\
\hline Avg. & - & - & - & - & - & 2571.38 & 57.51 & 645.47 & 1254.47 & - & \\
\hline
\end{tabular}

\#8 in Table 6). 


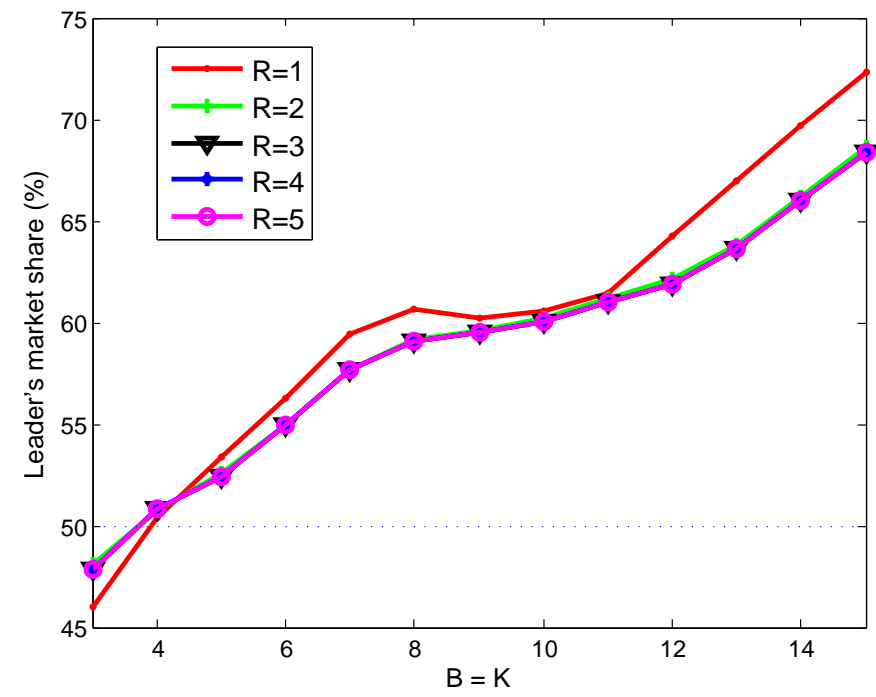

(a) leader's market share

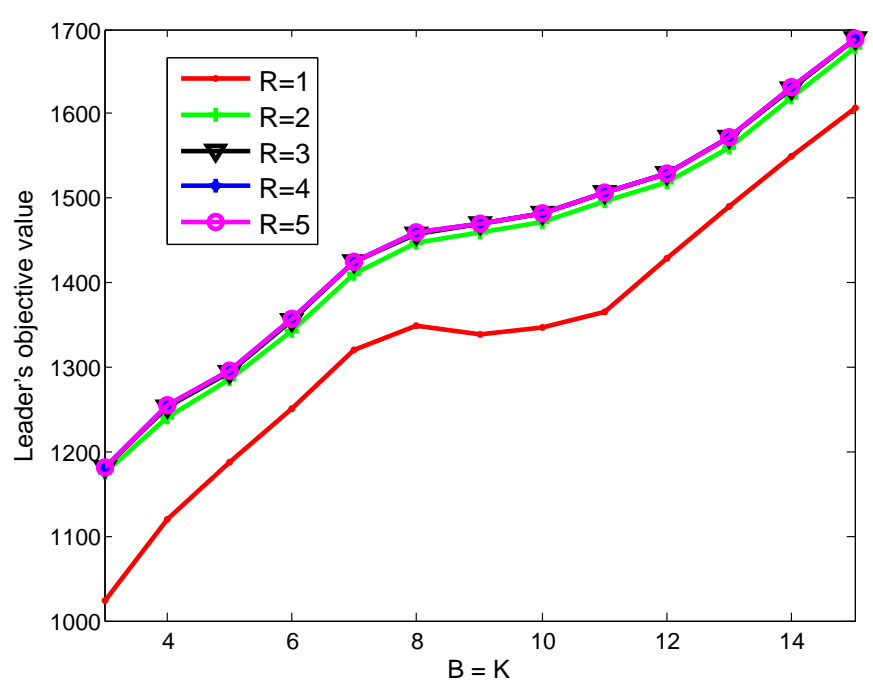

(b) leader's objective value

Figure 3: $|J|=30, q=0.1$

\subsubsection{Failure Probability $q$}

We again assume that both players open the same number of facilities $(B=K)$ and that this number is a constant. An interesting observation is that when they both open 5 facilities $(B=K=5$, \#1-\#6 in Table 6), the leader's market share will decrease as the failure probability $q$ increases, whereas when both of them open 4 facilities ( $B=K=4$, \#11-\#16 in Table 7), the leader's market share will increase as $q$ increases. This observation is further demonstrated in Figure 1, where all six curves intersect at the same point (approximately $B=K=4.4$ ). So if both players open a large number of facilities ( $B=K \geq 5)$, the leader will benefit from small $q$; in contrast, if they open a small number of facilities ( $B=K \leq 4)$, the leader will benefit from large $q$. This is because, when $q$ is large, the increased chance that customers will change their service location due to disruptions reduces the first-mover advantage in the leader's location selection. Therefore, the leader gains less benefit, even for large $B$.

\subsubsection{Maximum Assignment Level $R$}

The maximum assignment level $R$ can be explained as the maximum number of facilities that the customer is willing to seek for service. If all $R$ facilities that are closest to the customer are disrupted, the customer's demand is lost, so both players will lose that customer. In that sense, our model and solution algorithms can also suit the case in which each customer has a different tolerated number of facilities for service. Small $R$ indicates that the customers are conservative and can tolerate few backup 
service locations due to occasional disruptions, while large $R$ implies that the customers are flexible and can accept a large number of facilities for service.

As shown in Figure 3(a), if $B=K \geq 5$, the leader will benefit more from a market with conservative customers; while if $B=K \leq 4$, the leader will benefit from flexible customers. This conclusion is related to consumer behavior analysis. The same conclusions can be observed in \#7-\#11 in Table 6 and \#17-\#21 in Table 7. This is because, when $B$ is large, the leader is more likely to occupy all the good locations, better controlling the lower levels (especially the primary level, $r=1$ ); thus the leader favors conservative customers ( $R$ is small). Conversely, when $B$ is small, the leader favors flexible customers ( $R$ is large), who will change the firm they patronize due to disruptions; thus the leader has more opportunity to serve them on the backup level $(r \geq 2)$. In addition, from Table 6, we observe that the difference is not that significant between larger $R$ (e.g., $R=4$ and $R=5$ ); this is because of the scaling in the objective function (4), i.e., the demand captured decreases exponentially with $r$. The leader's objective value increases as the assignment level $R$ increases (Figure 3(b) ; this is because customers will have more choice in case of disruptions and so less demand will be lost.

\subsubsection{Number of Potential Facilities $|J|$}

From Figure 2, we observe that if the area that is available for the players to locate facilities in is limited $(|J|$ is small), the leader will benefit more from the smaller-failure-probability case. And the smaller $|J|$ is, the more beneficial it will be for the leader to dominate the market. That is also because of the first-mover advantage: The fewer potential facilities there are, the more possibility the leader will have to control the best locations.

The numerical results for the 88-node dataset are summarized in Table 8 . The results remain consistent with those from the 49-node dataset. Despite the increased problem sizes, the proposed solution approach can still solve most of these instances efficiently, within 10 minutes.

\subsection{Comparison of the Models: RPCP and CFLPD}

We are also interested in comparing the solutions that result from considering disruptions (i.e., reliable solutions (Snyder and Daskin 2005)) and from ignoring disruptions (i.e., non-reliable solutions), and the error that results if one were to ignore disruptions as a heuristic to solve a problem in which disruptions actually exist. To this end, we perform a series of numerical experiments and show the results in Table 9. 


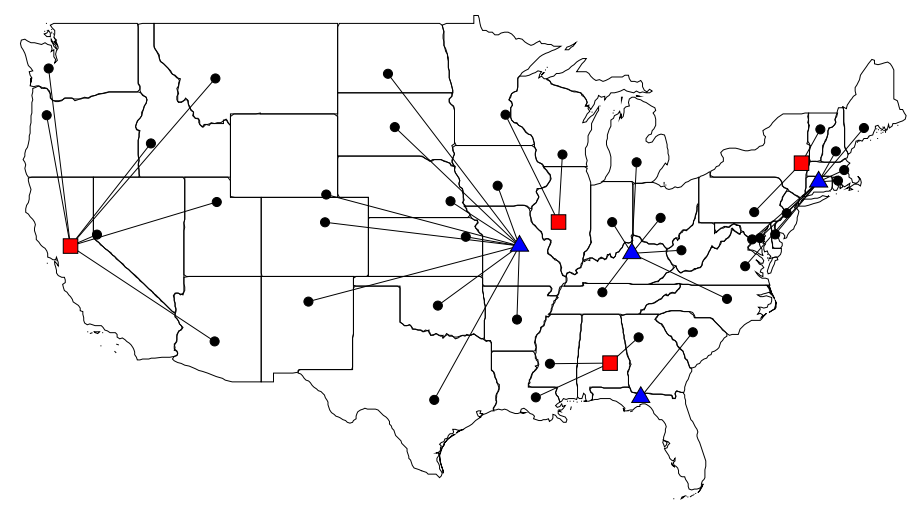

(a) non-reliable: $\operatorname{RPCP}(q=0)$

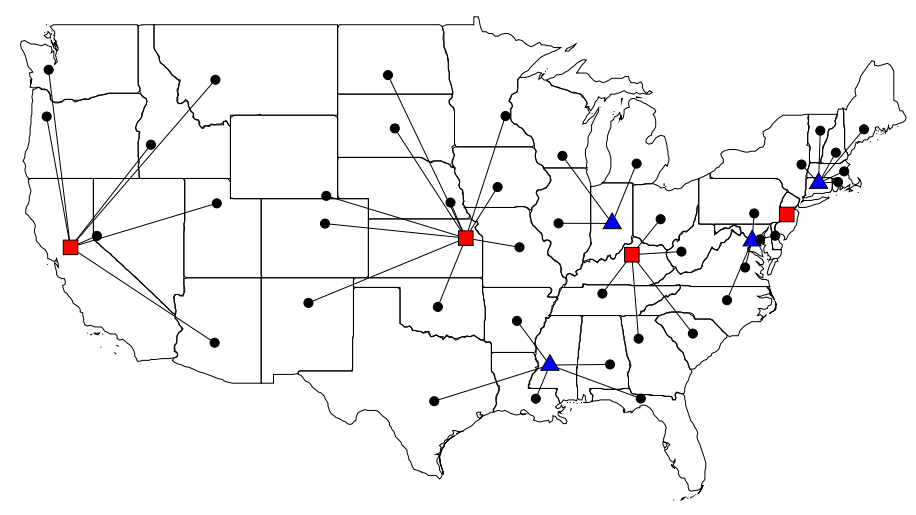

(c) reliable: \#13 $(q=0.06), \# 14(q=0.08), \# 15(q=0.10)$

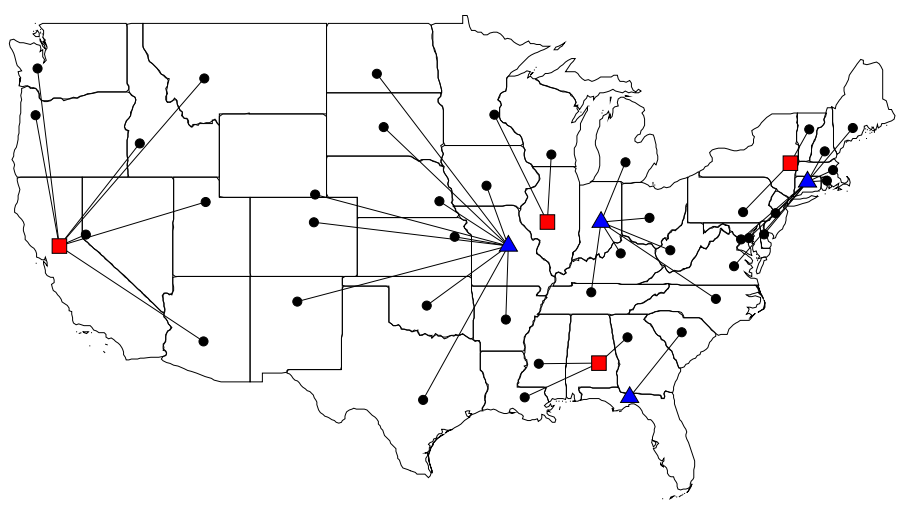

(b) reliable: \#11 $(q=0.02)$, \#12 $(q=0.04)$

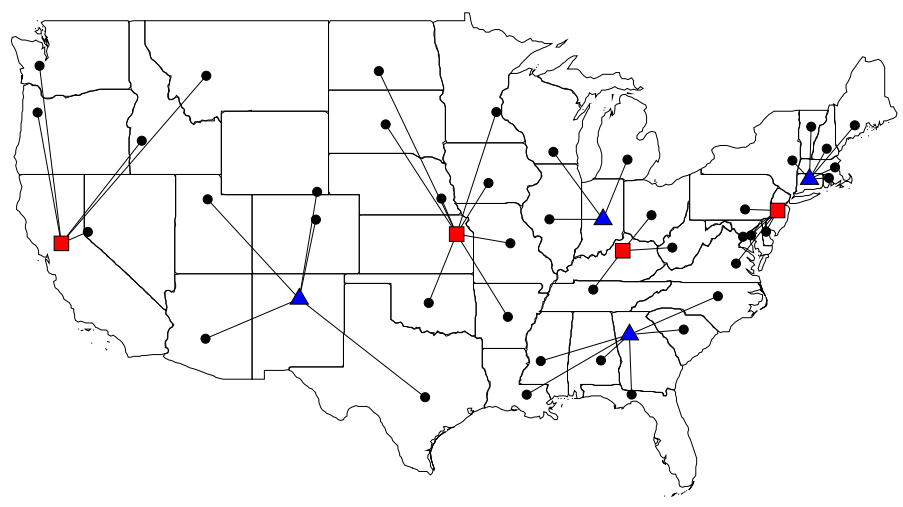

(d) reliable: \#16 $(q=0.20)$

Figure 4: Comparison $(|I|=|J|=49, B=K=4, R=3$; black circles: customers; red rectangles: leader's facilities; blue triangles: follower's facilities)

For each parameter setting in Table 7 , we solve a $\operatorname{RPCP}(q=0, R=1)$; once the algorithm terminates, the leader's objective value is recorded in the "LB1" column; the leader's and the follower's decisions are shown in the "Leader" column and the "Follower1" column, respectively; then, for the given leader's decision, the follower's problem when considering disruptions is solved exactly, the associated follower's decision is reported in the "Follower" column, the leader's true objective value under disruptions is shown in the "LB" column, and the percentage difference compared with the reliable case is listed in the "error" column. As expected, if solving the RPCP happens to yield the same leader's solution, then the leader's objective value will be the same as the reliable case considering disruptions. Tests \#11-\#21 from Table 9 show that an integer solution that is bilevel feasible in the RPCP model is not necessarily feasible in the CFLPD model, and if parameters change, for a given leader's solution, the follower may have different optimal responses. From Table 9, we observe that 
Table 9: Evaluation of RPCP solutions on CFLPD models $(|I|=|J|=49)$

\begin{tabular}{|c|c|c|c|c|c|c|c|c|c|c|c|c|}
\hline \multirow[t]{2}{*}{ No. } & \multirow[t]{2}{*}{$B$} & \multirow[t]{2}{*}{$K$} & \multirow[t]{2}{*}{$q$} & \multirow[t]{2}{*}{$R$} & \multirow{2}{*}{$\begin{array}{r}\text { Reliable } \\
\text { LB }\end{array}$} & \multicolumn{7}{|c|}{ Non-reliable } \\
\hline & & & & & & LB1 & time & Leader & Follower1 & Follower & LB & error \\
\hline 1 & 5 & 3 & 0.10 & 2 & 1554.70 & 1574.64 & 35.12 & $1,7,9,11,32$ & $19,37,42$ & $19,23,37$ & 1549.71 & 0.32 \\
\hline 2 & 5 & 4 & 0.10 & 2 & 1351.11 & 1383.53 & 50.21 & $1,2,6,22,28$ & $3,4,14,27$ & $3,5,14,36$ & 1338.34 & 0.95 \\
\hline 3 & 5 & 5 & 0.10 & 2 & 1207.16 & 1220.62 & 52.32 & $1,2,6,22,28$ & $3,4,14,27,36$ & $3,12,14,27,36$ & 1196.66 & 0.87 \\
\hline 4 & 5 & 6 & 0.10 & 2 & 1082.10 & 1082.59 & 50.82 & $1,2,6,7,22$ & $4,14,15,19,39,42$ & $4,15,23,27,39,47$ & 1082.10 & 0.00 \\
\hline 5 & 5 & 7 & 0.10 & 2 & 1001.92 & 989.63 & 60.23 & $1,2,6,7,22$ & $4,8,14,15,19,39,42$ & $4,5,15,20,23,27,39$ & 1001.92 & 0.00 \\
\hline 6 & 3 & 5 & 0.05 & 2 & 706.68 & 690.11 & 42.37 & $1,2,6$ & $14,15,39,42,45$ & $12,15,23,27,39$ & 706.68 & 0.00 \\
\hline 7 & 4 & 5 & 0.05 & 2 & 995.71 & 982.04 & 41.49 & $1,2,6,22$ & $4,15,23,27,39$ & $12,15,23,27,39$ & 995.71 & 0.00 \\
\hline 8 & 5 & 5 & 0.05 & 2 & 1216.88 & 1220.62 & 67.34 & $1,2,6,22,28$ & $3,4,14,27,36$ & $3,12,23,27,36$ & 1216.39 & 0.04 \\
\hline 9 & 6 & 5 & 0.05 & 2 & 1422.81 & 1427.63 & 64.29 & $1,2,6,7,22,28$ & $3,4,5,36,39$ & $3,4,15,19,39$ & 1422.81 & 0.00 \\
\hline 10 & 7 & 5 & 0.05 & 2 & 1593.97 & 1616.33 & 75.97 & $1,2,6,7,19,22,28$ & $3,4,5,36,39$ & $3,4,5,15,39$ & 1593.97 & 0.00 \\
\hline 11 & 4 & 4 & 0.02 & 3 & 1145.29 & 1143.09 & 54.95 & $1,2,6,22$ & $4,15,23,27$ & $4,14,15,27$ & 1145.29 & 0.00 \\
\hline 12 & 4 & 4 & 0.04 & 3 & 1147.13 & 1143.09 & 54.95 & $1,2,6,22$ & $4,15,23,27$ & $4,15,23,27$ & 1147.13 & 0.00 \\
\hline 13 & 4 & 4 & 0.06 & 3 & 1149.65 & 1143.09 & 54.95 & $1,2,6,22$ & $4,15,23,27$ & $12,15,23,27$ & 1144.66 & 0.43 \\
\hline 14 & 4 & 4 & 0.08 & 3 & 1161.32 & 1143.09 & 54.95 & $1,2,6,22$ & $4,15,23,27$ & $12,15,23,27$ & 1141.57 & 1.70 \\
\hline 15 & 4 & 4 & 0.10 & 3 & 1171.88 & 1143.09 & 54.95 & $1,2,6,22$ & $4,15,23,27$ & $12,15,23,27$ & 1138.35 & 2.86 \\
\hline 16 & 4 & 4 & 0.20 & 3 & 1199.89 & 1143.09 & 54.95 & $1,2,6,22$ & $4,15,23,27$ & $15,23,39,47$ & 1117.36 & 6.88 \\
\hline 17 & 4 & 4 & 0.05 & 1 & 1085.94 & 1143.09 & 54.95 & $1,2,6,22$ & $4,15,23,27$ & $4,14,15,27$ & 1085.94 & 0.00 \\
\hline 18 & 4 & 4 & 0.05 & 2 & 1144.80 & 1143.09 & 54.95 & $1,2,6,22$ & $4,15,23,27$ & $12,14,15,27$ & 1143.55 & 0.11 \\
\hline 19 & 4 & 4 & 0.05 & 3 & 1146.46 & 1143.09 & 54.95 & $1,2,6,22$ & $4,15,23,27$ & $12,15,23,27$ & 1146.17 & 0.03 \\
\hline 20 & 4 & 4 & 0.05 & 4 & 1146.51 & 1143.09 & 54.95 & $1,2,6,22$ & $4,15,23,27$ & $12,15,23,27$ & 1146.25 & 0.02 \\
\hline 21 & 4 & 4 & 0.05 & 5 & 1146.51 & 1143.09 & 54.95 & $1,2,6,22$ & $4,15,23,27$ & $12,15,23,27$ & 1146.26 & 0.02 \\
\hline Avg. & - & - & - & - & 1179.92 & 1179.13 & 54.51 & - & - & - & 1171.75 & 0.68 \\
\hline
\end{tabular}

for certain parameter settings, the RPCP can be a good heuristic. However, this may not be a safe approach to use, in general, since the RPCP model ignores possible disruptions. That is, except for some special cases, the RPCP solutions will not be accurate for the CFLPD, and instead one should use the proposed methods to solve the general problem.

Figure 4 shows the approximate facility deployments and the corresponding (level-1) customer assignments for different $q(|I|=|J|=49, B=K=4, R=3)$. In detail, Figure 4(a) shows the RPCP solution, as reported in \#11-\#21 from Table 9; Figures 4(b) 4(d) show CFLPD solutions for various $q$ as reported in \#11-\#16 from Table 7 . In the numerical analysis in $\$ 5.1$, we find that several different deployments may yield the same leader's objective value. However, we can always choose one that is more reliable. This is demonstrated in the comparison of Figure 4(a) and 4(b), One of the follower's facilities, Frankfort, KY, in Figure 4(a) is replaced by another facility, Indianapolis, IN, in Figure 4(b). The solutions in Figures 4(a) and 4(b) have the same leader' objective value, if evaluated under the RPCP model. But the solution in Figure 4(b) is more beneficial for the leader, since she will serve more customers at the backup level, gaining a larger market share if disruptions occur. Next, we observe that none of the customers are closest to the leader's facility in Trenton, NJ, in Figure 4(c). But it is still optimal for the leader to select this facility, since many customers are assigned to Trenton at level 2, if the follower's facilities in Hartford, CT and Washington, DC fail. Furthermore, we see that the optimal facilities tend to be more dispersed as the failure probability $q$ increases, as highlighted in the 
four figures in Figure 4 (as $q$ varies from 0 to 0.2 ). Intuitively, more dispersed facilities can provide better backups to each other in a competitive environment.

\section{Conclusions and Future Research}

In this paper we investigate a new discrete competitive facility location problem by taking into account the sequential game between two players and by considering facility disruption risks. The players successively place their facilities to capture customers, in order to maximize their profit. Customers seek the nearest functional facility for service. We formulate this problem as a binary bilevel linear optimization problem (BBLP) to determine the locations for the leader to maximize her market share, under the strongest possible response by the follower.

We then discuss the challenges associated with solving this problem. To solve it, we design a VNDS heuristic. The VNDS method combines, in a nested schema, hard variable fixing, soft variable fixing and linear constraints. Numerical studies are conducted first on a special case of our problem, the RPCP. The results demonstrate that the proposed VNDS method is very promising compared with the state-of-the-art heuristics and exact approaches from the literature. Then, we test the algorithm on CFLPD instances. Managerial insights are obtained through sensitivity analysis of the model and through several illustrative examples. For example, the player who locates more facilities will control most of the market. In the case in which the follower will react by locating the same number of facilities, the leader tends to dominate the market for large values of $B$ and $K$. In addition, if $B$ and $K$ are large ( $B=K \geq 5$ ), the leader will benefit from facilities that are more reliable ( $q$ is small) and conservative

customers who tolerate few backup locations ( $R$ is small); if $B$ and $K$ are small $(B=K \leq 4)$, the leader will benefit from vulnerable facilities ( $q$ is large) and flexible customers that can accept a large number of facilities for service ( $R$ is large). And the fewer the available potential locations are, the more beneficial it will be for the leader to dominate the market.

We believe that the proposed mathematical model is an important generalization of the classical facility location problem in terms of applications. This model has great potential for application where the decision on the placement of facilities is taken by two or more parties, and the facilities may fail from time to time. The VNDS matheuristic we design can be seen as a generalization of the familiar algorithm used for the solution of standard MIPs. The primary advantage of this approach is the ability to exploit the vast array of existing technology for solving MIPs techniques. All the usual rules of fathoming and branching are preserved. Our computational analysis shows that it is possible, 
in principle, to generalize the MIP matheuristics to solve BBLPs, a very challenging computational setting.

In the proposed CFLPD, we assume that the leader knows the exact number of facilities opened by the follower. In reality, the number of facilities could be flexible and it mainly depends on capital constraints. One future research topic is to develop stochastic or robust models to maximize the leader's market share under a range of scenarios specifying the number of follower's facilities. In addition, from a practical perspective, it is also important to generalize the assumption of uncapacitated facilities and identical failure probabilities. Furthermore, future research can be focused on the development of improved bound to achieve global optimality, and the development of more efficient computational methods.

\section{Acknowledgments}

This work was supported by the National Natural Science Foundation of China (71502112), and partly sponsored by the State Scholarship Fund of China Scholarship Council. In addition, this research was supported in part by NSF grant \#CMMI-1541177 and will contribute to the PRAISys platform (www. praisys.org). This support is gratefully acknowledged. We also wish to thank the anonymous referees for their helpful suggestions, which led to improved clarity of the paper and improved computational performance of the algorithms.

\section{References}

Aboolian, Robert, Tingting Cui, Zuo-Jun Max Shen. 2012. An efficient approach for solving reliable facility location models. INFORMS Journal on Computing 25(4) 720-729.

Aksen, Deniz, Sema Şengül Akca, Necati Aras. 2014. A bilevel partial interdiction problem with capacitated facilities and demand outsourcing. Computers \& Operations Research 41 346-358.

Alekseeva, E, N Kochetova, Yu Kochetov, A Plyasunov. 2009. A hybrid memetic algorithm for the competitive p-median problem. Preprints of the 13th IFAC Symposium on Information Control Problems in Manufacturing, Moscow. 1516-1520.

Alekseeva, Ekaterina, Yury Kochetov. 2013. Matheuristics and exact methods for the discrete $(r \mid p)$-centroid problem. Metaheuristics for Bi-Level Optimization. Springer, 189-219.

Alekseeva, Ekaterina, Nina Kochetova, Yury Kochetov, Alexandr Plyasunov. 2010. Heuristic and exact methods for the discrete $(r \mid p)$-centroid problem. Evolutionary Computation in Combinatorial Optimization. Springer, 11-22. 
Ashtiani, Milad Gorji, Ahmad Makui, Reza Ramezanian. 2013. A robust model for a leadercfollower competitive facility location problem in a discrete space. Applied Mathematical Modelling 37(1C2) 62 - 71 . doi: http://dx.doi.org/10.1016/j.apm.2011.12.013. URL http://www.sciencedirect.com/science/article/ pii/S0307904X11007992.

Audet, Charles, Gilles Savard, Walid Zghal. 2007. New branch-and-cut algorithm for bilevel linear programming. Journal of Optimization Theory and Applications 134(2) 353-370.

Berman, Oded, Dmitry Krass, Mozart BC Menezes. 2007. Facility reliability issues in network p-median problems: strategic centralization and co-location effects. Operations Research 55(2) 332-350.

Bialas, Wayne F, Mark H Karwan. 1984. Two-level linear programming. Management Science 30(8) 1004-1020.

Biesinger, Benjamin, Bin Hu, Günther Raidl. 2015. A hybrid genetic algorithm with solution archive for the discrete $(r \mid p)$-centroid problem. Journal of Heuristics 21(3) 391-431.

Bixby, E Robert, Mary Fenelon, Zonghao Gu, Ed Rothberg, Roland Wunderling. 2000. Mip: Theory and practiceclosing the gap. System Modelling and Optimization. Springer, 19-49.

Church, Richard, Charles R Velle. 1974. The maximal covering location problem. Papers in Regional Science 32(1) 101-118.

Cui, Tingting, Yanfeng Ouyang, Zuo-Jun Max Shen. 2010. Reliable facility location design under the risk of disruptions. Operations Research 58(4-part-1) 998-1011.

Danna, Emilie, Edward Rothberg, Claude Le Pape. 2005. Exploring relaxation induced neighborhoods to improve mip solutions. Mathematical Programming 102(1) 71-90.

Daskin, Mark S. 2013. Network and Discrete Location: Models, Algorithms, and Applications. 2nd ed. Wiley, New York.

Davydov, Ivan Aleksandrovich. 2012. Local tabu search for the discrete $(r \mid p)$-centroid problem. Diskret. Anal. Issled. Oper. 19(2) 19-40.

Davydov, Ivan Aleksandrovich, Yu A Kochetov, Nenad Mladenovic, D Urosevic. 2014. Fast metaheuristics for the discrete $(r \mid p)$-centroid problem. Automation and Remote Control 75(4) 677-687.

Dempe, Stephan. 2002. Foundations of bilevel programming. Springer Science \& Business Media.

DeNegre, ST, Ted K Ralphs. 2009. A branch-and-cut algorithm for integer bilevel linear programs. Operations Research and Cyber-Infrastructure. Springer, 65-78.

Eiselt, Horst A, Gilbert Laporte. 1997. Sequential location problems. European Journal of Operational Research 96(2) 217-231.

Eiselt, Horst A, Gilbert Laporte, Jacques-Francois Thisse. 1993. Competitive location models: A framework and bibliography. Transportation Science 27(1) 44-54.

Farahani, Reza Zanjirani, Shabnam Rezapour, Tammy Drezner, Samira Fallah. 2014. Competitive supply chain 
network design: An overview of classifications, models, solution techniques and applications. Omega $\mathbf{4 5}$ $92-118$.

Fischetti, Matteo, Andrea Lodi. 2003. Local branching. Mathematical Programming 98(1-3) 23-47.

Gedik, Ridvan, Hugh Medal, Chase Rainwater, Ed A Pohl, Scott J Mason. 2014. Vulnerability assessment and re-routing of freight trains under disruptions: A coal supply chain network application. Transportation Research Part E: Logistics and Transportation Review 71 45-57.

Hanafi, Saïd, Jasmina Lazić, Nenad Mladenović, Christophe Wilbaut, Igor Crévits. 2014. New variable neighbourhood search based 0-1 mip heuristics. Yugoslav Journal of Operations Research (00) 14-14.

Hansen, Pierre, Nenad Mladenović. 2001. Variable neighborhood search: Principles and applications. European Journal of Operational Research 130(3) 449-467.

Hansen, Pierre, Nenad Mladenović, José A Moreno Pérez. 2010. Variable neighbourhood search: methods and applications. Annals of Operations Research 175(1) 367-407.

Hansen, Pierre, Nenad Mladenović, Dionisio Perez-Britos. 2001. Variable neighborhood decomposition search. Journal of Heuristics 7(4) 335-350.

Hansen, Pierre, Nenad Mladenović, Dragan Urošević. 2006. Variable neighborhood search and local branching. Computers \& Operations Research 33(10) 3034-3045.

Kochetov, Yury, Nina Kochetova, Alexandr Plyasunov. 2013. A matheuristic for the leader-follower facility location and design problem. Proceedings of the 10th Metaheuristics International Conference (MIC 2013). 32.

Kress, Dominik, Erwin Pesch. 2012. Sequential competitive location on networks. European Journal of Operational Research 217(3) 483-499.

Lazić, Jasmina, Saïd Hanafi, Nenad Mladenović, Dragan Urošević. 2010. Variable neighbourhood decomposition search for 0-1 mixed integer programs. Computers \& Operations Research 37(6) 1055-1067.

Liberatore, Federico, Maria P Scaparra, Mark S Daskin. 2011. Analysis of facility protection strategies against an uncertain number of attacks: the stochastic r-interdiction median problem with fortification. Computers \& Operations Research 38(1) 357-366.

Losada, Chaya, M Paola Scaparra, Jesse R O' Hanley. 2012. Optimizing system resilience: a facility protection model with recovery time. European Journal of Operational Research 217(3) 519-530.

Moore, James T, Jonathan F Bard. 1990. The mixed integer linear bilevel programming problem. Operations Research 38(5) 911-921.

Noltemeier, Hartmut, Joachim Spoerhase, H-C Wirth. 2007. Multiple voting location and single voting location on trees. European Journal of Operational Research 181(2) 654-667.

O' Hanley, Jesse R, Richard L Church. 2011. Designing robust coverage networks to hedge against worst-case facility losses. European Journal of Operational Research 209(1) 23-36. 
Plastria, Frank, Lieselot Vanhaverbeke. 2008. Discrete models for competitive location with foresight. Computers \& Operations Research 35(3) 683-700.

Roboredo, Marcos Costa, Artur Alves Pessoa. 2013. A branch-and-cut algorithm for the discrete $(r \mid p)$-centroid problem. European Journal of Operational Research 224(1) 101-109.

Serra, Daniel, Charles ReVelle. 1994. Market capture by two competitors: The preemptive location problem. Journal of Regional Science 34(4) 549-561.

Serra, Daniel, Charles ReVelle, et al. 1994. Competitive location in discrete space. Universitat Pompeu Fabra.

Seyhan, Tolga H., Lawrence V. Snyder, Ying Zhang. 2015. A new heuristic formulation for a competitive maximal covering location problem .

Shen, Zuo-Jun Max, Roger Lezhou Zhan, Jiawei Zhang. 2011. The reliable facility location problem: Formulations, heuristics, and approximation algorithms. INFORMS Journal on Computing 23(3) 470-482.

Snyder, Lawrence V. 2010. Introduction to facility location. Wiley Encyclopedia of Operations Research and Management Science .

Snyder, Lawrence V, Zümbül Atan, Peng Peng, Ying Rong, Amanda J Schmitt, Burcu Sinsoysal. 2015. Or/ms models for supply chain disruptions: A review. IIE Transactions 1-21.

Snyder, Lawrence V, Mark S Daskin. 2005. Reliability models for facility location: the expected failure cost case. Transportation Science 39(3) 400-416.

Wang, Xin, Yanfeng Ouyang. 2013. A continuum approximation approach to competitive facility location design under facility disruption risks. Transportation Research Part B: Methodological 50 90-103.

Zhang, Ying, Lawrence V Snyder, Mingyao Qi, Lixin Miao. 2016. A heterogeneous reliable location model with risk pooling under supply disruptions. Transportation Research Part B: Methodological 83 151-178. 


\section{The competitive facility location problem under disruption risks}

\section{Ying Zhang}

Research Center on Modern Logistics, Graduate School at Shenzhen, Tsinghua University, Shenzhen 518055, China

Department of Industrial Engineering, Tsinghua University, Beijing 100084, China

Email: yingzhang8996@gmail.com

Lawrence V. Snyder

Department of Industrial and Systems Engineering, Lehigh University, 200 West Packer Ave., Mohler Lab, Bethlehem, PA 18015, USA

Email: larry.snyder@lehigh.edu

Ted K. Ralphs

Department of Industrial and Systems Engineering, Lehigh University, 200 West Packer Ave., Mohler Lab, Bethlehem, PA 18015, USA

Email: ted@lehigh.edu 